\title{
An experimental exploration of the properties of random frequency response functions
}

\author{
A. Clot $^{1 *}$, J.W.R. Meggitt ${ }^{2}$, R.S. Langley ${ }^{1}$, A.S. Elliott ${ }^{2}$, A.T. Moorhouse ${ }^{2}$ \\ ${ }^{1}$ Department of Engineering, University of Cambridge \\ ${ }^{2}$ Acoustics Research Centre, University of Salford
}

\begin{abstract}
The vibro-acoustic analysis of complex structures over a broadband frequency range is an extremely challenging problem that may often require the use of a hybrid deterministic-statistical approach. Due to manufacturing imperfections, the frequency response functions (FRFs) of an ensemble of nominally identical systems can be considered to be random. These FRFs, however, have statistical properties that can be potentially used in vibro-acoustic models. This work explores some of these fundamental properties by using measured FRFs from an ensemble of nominally identical structures, obtained by randomising a thin rectangular plate using point masses. It is first shown that the measured ensemble of FRFs satisfies the analyticity-ergodicity condition, experimentally verifying this recently demonstrated fundamental property. Then, the ensemble is used to explore whether the direct field dynamic stiffness, a key parameter in a well-established hybrid deterministic-statistical formulation, can be obtained experimentally. The results are compared against those computed using numerical techniques, showing that measured data may be a suitable alternative provided that an ensemble of systems can be measured. Finally, an alternative method, based on the use of virtual point masses, opposed to physical ones, is proposed for those cases where experimental randomisation is particularly challenging. It has been found, however, that the method may be extremely sensitive to measurement imprecisions, specially when applied to lightly damped structures. It is concluded that the statistical properties of random causal FRFs are not only interesting in themselves, but can enhance and extend vibro-acoustic prediction models.
\end{abstract}

Keywords: Random frequency response functions; Experimental ensembles; Analyticity-ergodicity condition; Direct field dynamic stiffness

\section{Introduction}

The two main challenges that Finite Element (FE) models face when analysing the vibro-acoustic response of complex systems at high frequencies are (i) that an unreasonably large number of degrees of

${ }^{*}$ Corresponding author. E-mail address: arnau.clot@upc.edu. Present afiliation: Serra Húnter Fellow at Universitat Politècnica de Catalunya, Spain

Preprint submitted to Journal of Sound and Vibration

October 5, 2020 
freedom may be needed to represent the dynamic system accurately, and (ii) that the response of the system may be highly sensitive to small manufacturing imperfections. Both difficulties may be overcome by Statistical Energy Analysis (SEA), which provides a prediction of the mean response [1] and variance [1, 2] of an ensemble of nominally identical systems. A key advantage of SEA is that it requires only a small number of degrees of freedom, representing the vibrational energies of the subsystems, to model the entire vibro-acoustic system. Additional challenges arise when considering what is commonly referred as the midfrequency problem, i.e., the range of frequencies where FE and SEA approaches are not suitable to model all the components of system. A solution to this problem was proposed by Shorter and Langley [3], who presented a hybrid FE-SEA formulation based on a diffuse field reciprocity result [4, 5]. The method considers that the complex system is divided into a deterministic component (the master system) and a set of statistical components coupled together via the deterministic one. The hybrid method has been numerically and experimentally validated [6] and has been extended to predict the ensemble variance of the response 7]. More recently, parametric uncertainty has been included on the deterministic components of the system [8, 9].

The use of experimental data to extend the capabilities of hybrid FE-SEA models was recently studied in [10]. The work develops a case study which includes complex vibration sources that have been represented using experimental blocked forces [11, and resilient elements that have been experimentally characterised [12. In the presented case studies, an analytical method has been used to represent the point junctions between the deterministic (i.e. the experimental components) and SEA subsystems. Several analytical and numerical techniques have been considered for different types of simple junctions, including: point [13], line 6] and area [14 connections. This work presents an alternative method to determine the properties of a junction, based on the use of statistical properties of an ensemble of random systems.

The manufacturing variability of complex engineering systems suggest that vibration frequency response functions (FRFs) can be considered as random functions over an ensemble of systems [15]. The statistical properties of these complex FRF functions have been a question of interest for several authors. Lyon investigated the variance of their modulus [16] and the statistics of their phase [17, and Skudrzyk [18] and Cremer and Heckl [19] studied their mean value. More recently, Langley [20] showed that, under broad conditions, a complex FRF satisfies the analyticity-ergodicity (AE) condition, and presents extensive numerical evidence of this result. The AE condition had been previously considered by Mello et al. 21] in the context of random scattering matrices in nuclear physics [22]. An example of a related recent contribution is the work of Nock et al. [23], who obtained the probability density function of the real and imaginary components of an off-diagonal element of a scattering matrix. The work presented in [20] showed that the AE requirement considered in random scattering matrices can be also applied to vibrational FRFs. The validity of the $\mathrm{AE}$ condition is revisited in this work, which presents further experimental evidence of this result. 
The aims of this work are (i) to perform an experimental exploration of certain fundamental statistical properties of random FRFs, and (ii) to use these properties to extend the capabilities of the hybrid FE-SEA method. The remainder of this paper will be organised as follows: Section 2 describes the experimental set-up used in the work; The data obtained with this set-up is used in Section 3 to asses the validity of the AE condition, and to characterise experimentally the junctions between deterministic and statistical subsystems; A methodology to overcome the limitations that can be encountered when measuring an ensemble of subsystems is discussed in Section 4. Finally, Section 5 summarises the main conclusions of this work.

\section{Experimental set-up}

In this work the properties of experimental random frequency response functions are studied using an ensemble of "nominally identical" plates. This ensemble has been obtained by randomising experimentally a thin rectangular aluminium plate with dimensions $0.8 \mathrm{~m}$ (length) $\times 1 \mathrm{~m}$ (width) $\times 3 \mathrm{~mm}$ (thickness). Free boundary conditions have been approximately obtained by adding elastomeric pads along two parallel edges of the plate, leaving the remaining edges free. An ensemble of experimental systems has been obtained by adding a set of point masses to the plate, placed at randomly chosen locations considering (i) a minimum distance between them, (ii) a minimum distance between the masses and the measuring positions, and (iii) a minimum distance between the masses and the plate edges. A total of 11 masses were connected to the plate using magnetic bases. The total mass added was $800 \mathrm{~g}$, which corresponds to approximately $12 \%$ of the initial mass of the plate.

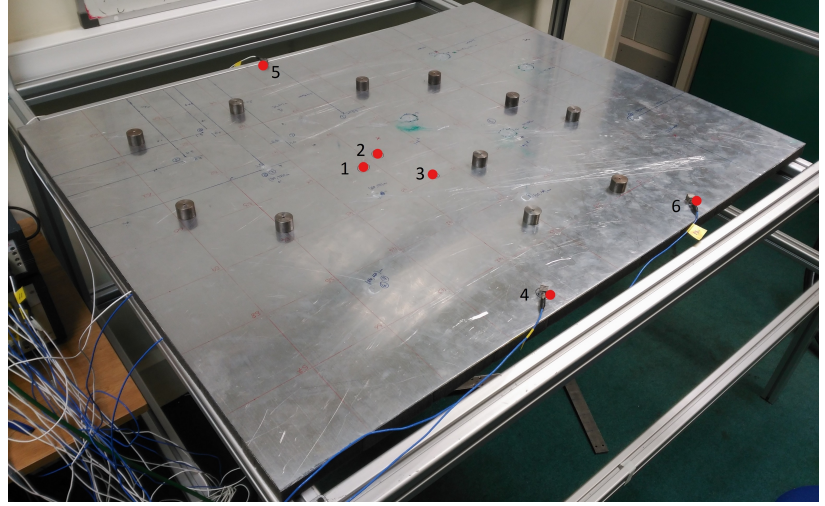

(a) Experimental set-up for a member of the ensemble of random plates. Measuring positions are marked with red dots.

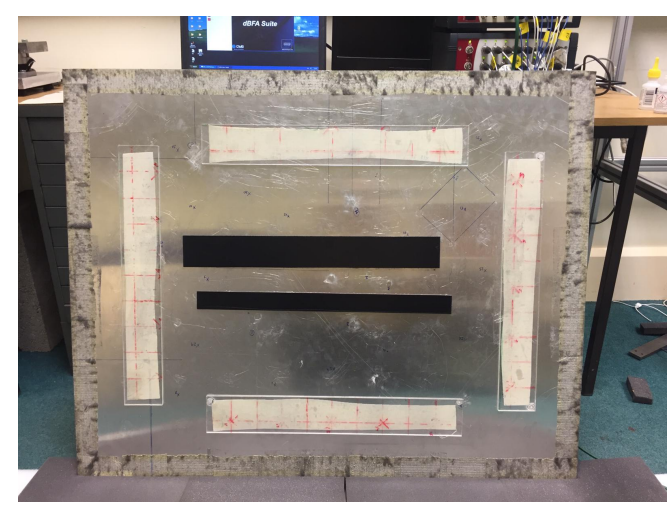

(b) Damping treatment added to the plate for the second ensemble of systems.

Figure 1: Experimental set-up for building an ensemble of random plates

The mechanical parameters considered for the aluminium were; density $\rho=2700 \mathrm{~kg} \cdot \mathrm{m}^{-3}$, Young modulus $E=70 \mathrm{GPa}$, and Poisson ratio $\nu=0.33$. The modal density was computed using the asymptotic expression for the bending modes of a thin plate [1] $n=L_{1} L_{2} / 4 \pi \sqrt{\rho h / D_{p}}$, giving $n=0.013 \operatorname{modes} /(\mathrm{rad} / \mathrm{s})$. The loss 
factor of the plate was determined experimentally, and a frequency-averaged value $\eta=0.8 \%$ was obtained over the range of frequencies considered $(1-5000 \mathrm{~Hz})$. With these values, the modal overlap factor of the plate at $1000 \mathrm{~Hz}$ is $m=\omega n \eta=0.67$.

The dynamic response of the plate was measured at six different positions, marked with red dots in Figure 1a, using accelerometers. They correspond to three points far from the plate edges, referred as interior points, and three points close to a plate edge, referred as near-edge points. The set of results were obtained by applying impact excitations at each one of these positions using an instrumented hammer, and measuring the response at all six positions. An accelerance matrix was obtained by dividing the measured acceleration spectra by the measured force spectrum for each excitation. An ensemble of random systems was obtained by repeating this test 20 times, with different mass locations each time.

A second ensemble was considered by applying a damping treatment on the plate structure. As it is shown in Figure $1 \mathrm{~b}$ the treatment consisted in several perspex strips glued to the bottom of the plate. As before, the loss factor of the plate was experimentally determined, obtaining a frequency-averaged value of $1.45 \%$. In this case, the modal overlap factor at $1000 \mathrm{~Hz}$ is $m=1.22$. An experimental ensemble of damped plates was obtained using the method described above. The same accelerometer positions were considered in this case.

\section{Results obtained using experimental ensembles}

In this section, the experimental ensembles obtained in Section 2 have been used to, first, verify that AE condition [20] is satisfied, and second, to explore the possibility of obtaining an experimental direct field dynamic stiffness [3].

\subsection{Analyticity-ergodicity condition}

In nuclear physics the $\mathrm{AE}$ condition states that $\mathrm{E}[f(\mathbf{H})]=f(\mathrm{E}[\mathbf{H}])$, where $\mathbf{H}$ is a random scattering matrix, $f(\quad)$ is some function of $\mathbf{H}$ and $\mathrm{E}[]$ represents an ensemble average. It is demonstrated in [20] that the AE condition is also applicable to a random causal FRF $\mathbf{H}$ if the following broad conditions are satisfied:

- The statistics of the natural frequencies of the system (poles) are described by a point process that is, at least, stationary for those natural frequencies that are close to the excitation frequency. This condition does not require the natural frequency distribution to conform to a universal distribution such as the Gaussian Orthogonal Ensemble (GOE) or the Poisson distribution [24].

- The function $f(\mathbf{H})$ must have a convergent Taylor series expansion, a condition that is likely to be met by the causal $f(\mathbf{H})$ and, therefore, analytic in the lower half-plane. 
The applicability of the AE condition to random causal FRFs was numerically verified in 20]. The aim of this section is to complement these numerical verifications with experimental ones, using the set of experimental ensembles described in Section 2, This aim is achieved by considering that the measured accelerance matrix $\mathbf{A}$ is the random causal FRF matrix considered, i.e. $\mathbf{H}=\mathbf{A}$, and comparing $\mathrm{E}[f(\mathbf{A})]$ with $f(\mathrm{E}[\mathbf{A}])$ for a given $f()$.

Figure 2 tests the validity of the AE condition for the case where $f(\mathbf{A})=\mathbf{A}^{-1}$, i.e., the function used is the matrix inverse performed to determine the apparent mass matrix. The experimental results used are the ones obtained by randomising the plate without added damping. The figure compares two components of the apparent mass matrices obtained by computing $\mathrm{E}\left[\mathbf{A}^{-1}\right]$ and $\mathbf{E}[\mathbf{A}]^{-1}$. The variability of the apparent mass over the ensemble is also included by plotting $\mathbf{A}^{-1}$ for each member of the experimental ensemble. The results show that the AE condition is clearly satisfied for most of the range of frequencies considered, and for both driving (a) and transfer (b) components of the apparent mass matrix. The results also show a significant ensemble variance of the apparent mass, illustrating that the system response is sufficiently random over the ensemble. The results also show that, for a small set of frequencies, the result obtained by averaging the apparent mass matrix (in blue) is considerably noisier than the one obtained by inverting the ensemble average of the acceleration matrix (in red). This important result, which suggest a potential benefit of using the $\mathrm{AE}$ condition, will be discussed in more detail in later sections.

(a)

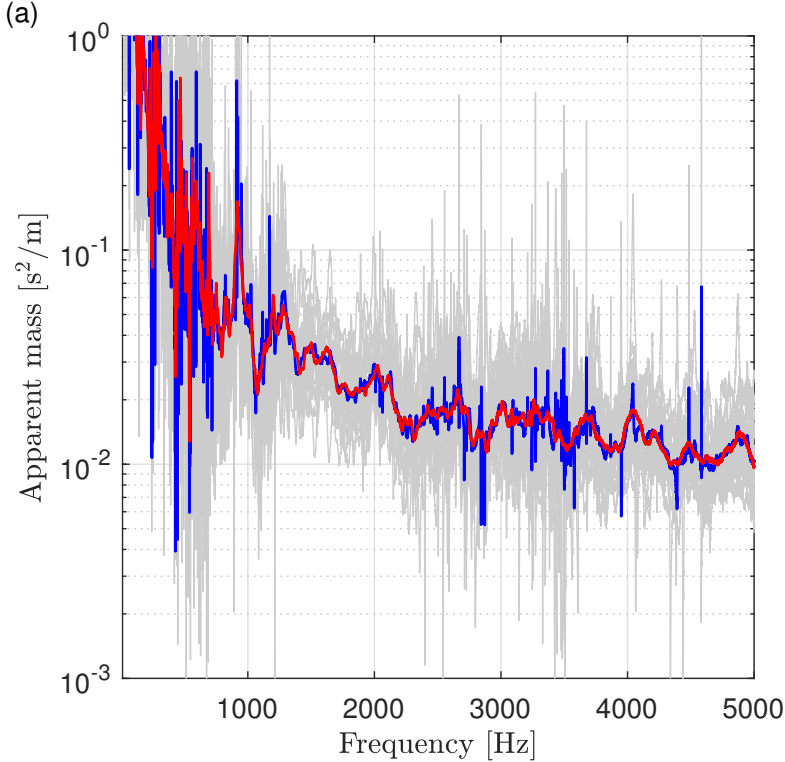

(b)

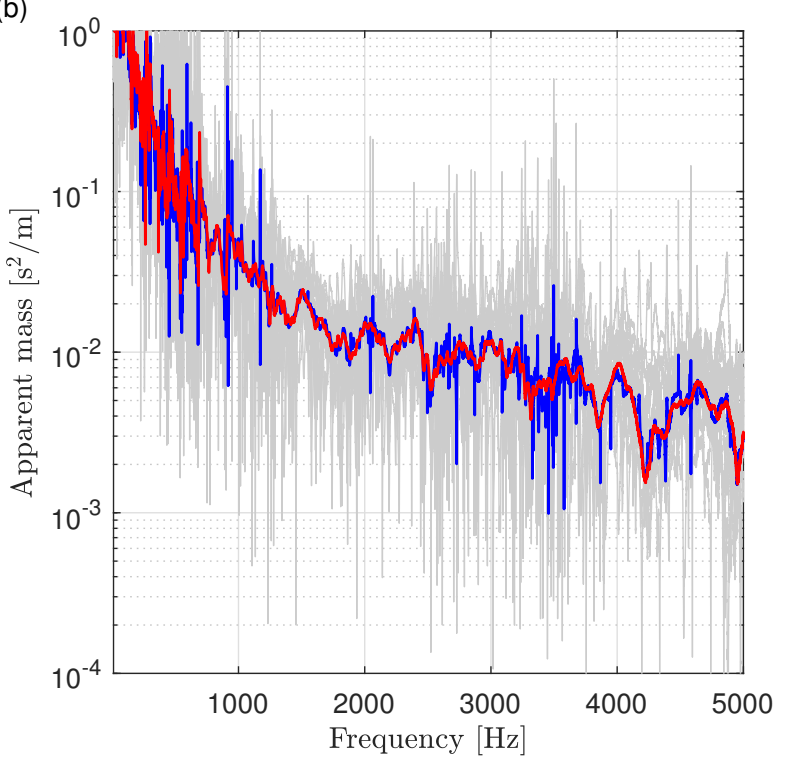

Figure 2: Experimental verification of the AE condition using the accelerance matrix of the plate without added damping, and with $f(\mathbf{A})=\mathbf{A}^{-1}$. (a) Modulus of $\mathrm{E}\left[\left(\mathbf{A}^{-1}\right)_{11}\right]$ (blue), $\left(\mathrm{E}[\mathbf{A}]^{-1}\right)_{11}$ (red) and $\left(\mathbf{A}^{-1}\right)_{11}$ (gray). (b) Modulus of $\mathrm{E}\left[\left(\mathbf{A}^{-1}\right)_{12}\right]$ (blue), $\left(\mathrm{E}[\mathbf{A}]^{-1}\right)_{12}$ (red) and $\left(\mathbf{A}^{-1}\right)_{12}$ (gray).

The robustness of the $\mathrm{AE}$ condition is studied in more detail in Figure 3, which shows the relative 


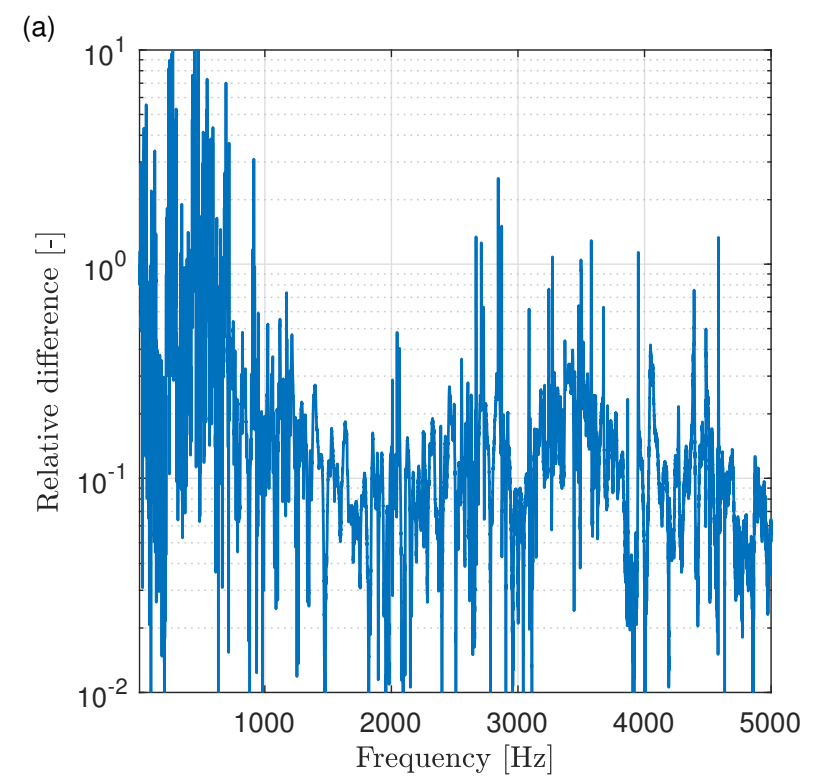
validity of the $\mathrm{AE}$ condition. (b)

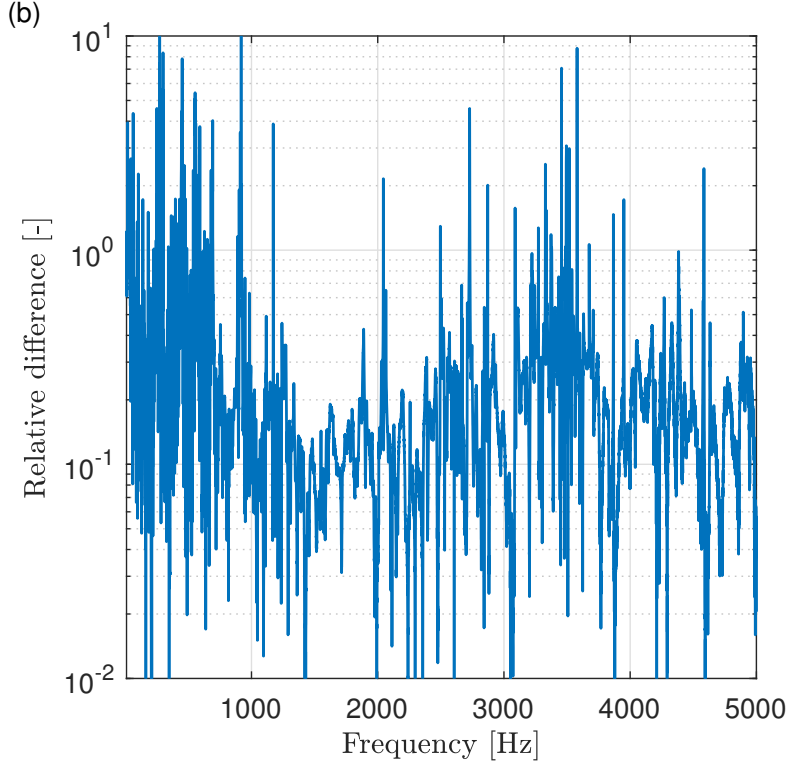

Figure 3: Relative difference in the AE condition for the case of a plate without added damping and $f(\mathbf{A})=\mathbf{A}^{-1}$. (a) Relative difference $\left|\left(E\left[\mathbf{A}^{-1}\right]-\mathrm{E}[\mathbf{A}]^{-1}\right)_{11}\right| /\left|\left(\mathrm{E}\left[\mathbf{A}^{-1}\right]\right)_{11}\right|$. (b) Relative difference $\left|\left(\mathrm{E}\left[\mathbf{A}^{-1}\right]-\mathrm{E}[\mathbf{A}]^{-1}\right)_{12}\right| /\left|\left(\mathrm{E}\left[\mathbf{A}^{-1}\right]\right)_{12}\right|$

difference, computed as $\left|\left(\mathrm{E}\left[\mathbf{A}^{-1}\right]-\mathrm{E}[\mathbf{A}]^{-1}\right)_{i j}\right| /\left|\left(\mathrm{E}\left[\mathbf{A}^{-1}\right]\right)_{i j}\right|$, for the two apparent mass components used in the previous figure. The results show that, in general, the AE condition is clearly satisfied at high frequencies. This result is in agreement with the fact that at low frequencies the amount of randomness added to the structure is insufficient to ensure that the statistics of the natural frequencies over the ensemble can be represented by a random point process 20. This is one of the two conditions required to ensure the

The role that the modal overlap $m$ plays in the validity of the AE condition can be studied using the experimental results obtained for the damped plate ensemble (see Figure 1b). The validity of the AE condition for $f(\mathbf{A})=\mathbf{A}^{-1}$ using the damped case data is presented in Figure 4 . As before, the variability of the apparent mass over the ensemble has been also included in the figure. The results show again that the AE condition is satisfied for both driving (a) and transfer (b) components of the apparent mass matrix. As expected, the higher damping on the system results in a smaller ensemble variance of the apparent mass. 

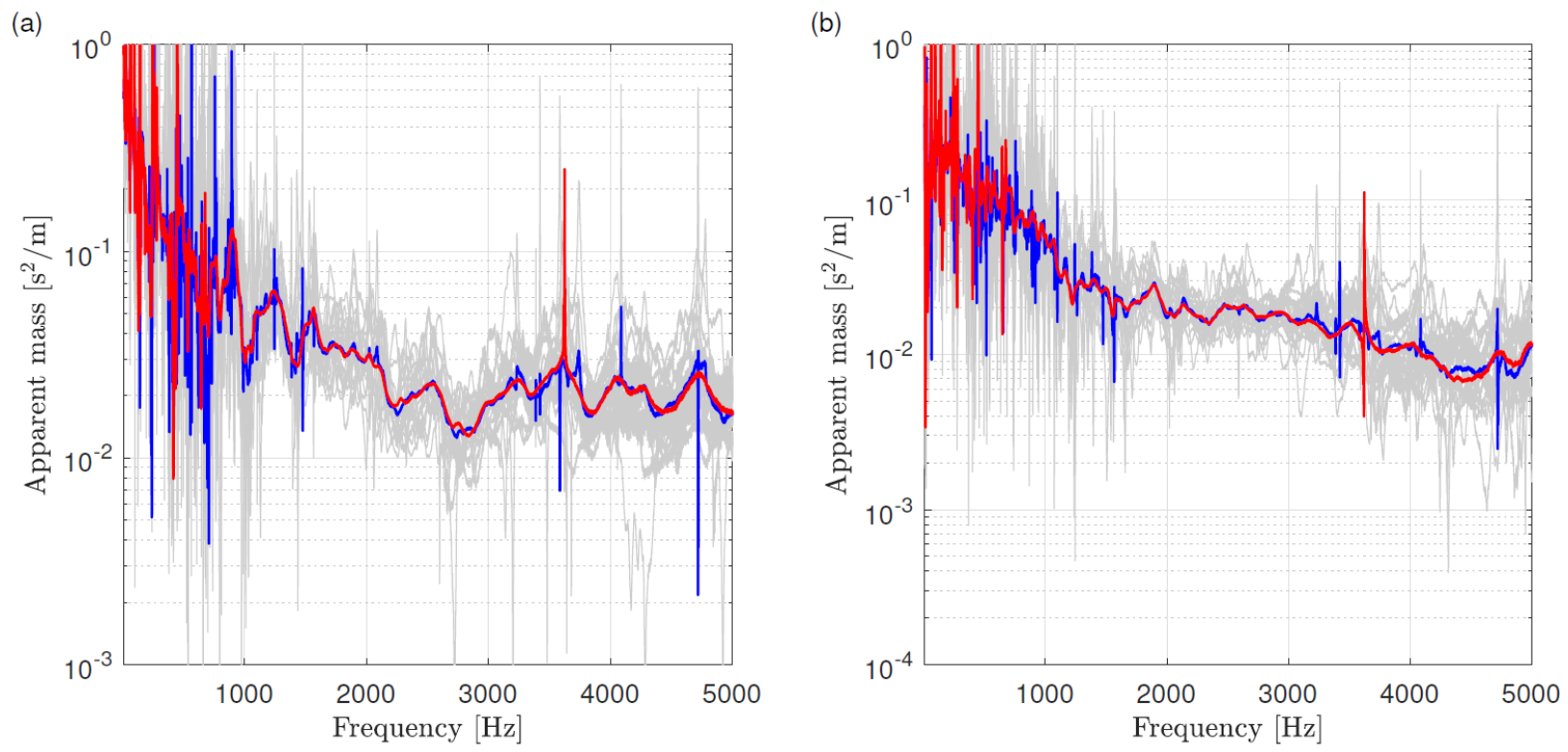

Figure 4: Experimental verification of the AE condition using the accelerance matrix of the plate with added damping, and with $f(\mathbf{A})=\mathbf{A}^{-1}$. (a) Modulus of $\mathrm{E}\left[\left(\mathbf{A}^{-1}\right)_{11}\right]$ (blue), $\left(\mathrm{E}[\mathbf{A}]^{-1}\right)_{11}$ (red) and $\left(\mathbf{A}^{-1}\right)_{11}$ (gray). (b) Modulus of $\mathrm{E}\left[\left(\mathbf{A}^{-1}\right)_{12}\right]$ (blue), $\left(\mathrm{E}[\mathbf{A}]^{-1}\right)_{12}$ (red) and $\left(\mathbf{A}^{-1}\right)_{12}$ (gray).

Figure 5 shows the relative difference $\left|\left(\mathrm{E}\left[\mathbf{A}^{-1}\right]-\mathrm{E}[\mathbf{A}]^{-1}\right)_{i j}\right| /\left|\left(\mathrm{E}\left[\mathbf{A}^{-1}\right]\right)_{i j}\right|$ for the case where $\mathbf{A}$ is the accelerance matrix of the plate with added damping. As before, the results show that the $\mathrm{AE}$ condition is clearly satisfied at high frequencies. The results also show that a larger modal overlap slightly smooths the relative difference, but does not have a significant effect in its frequency content. This result is also in agreement with the analysis presented in [20, which does not explicitly employ the modal overlap. 

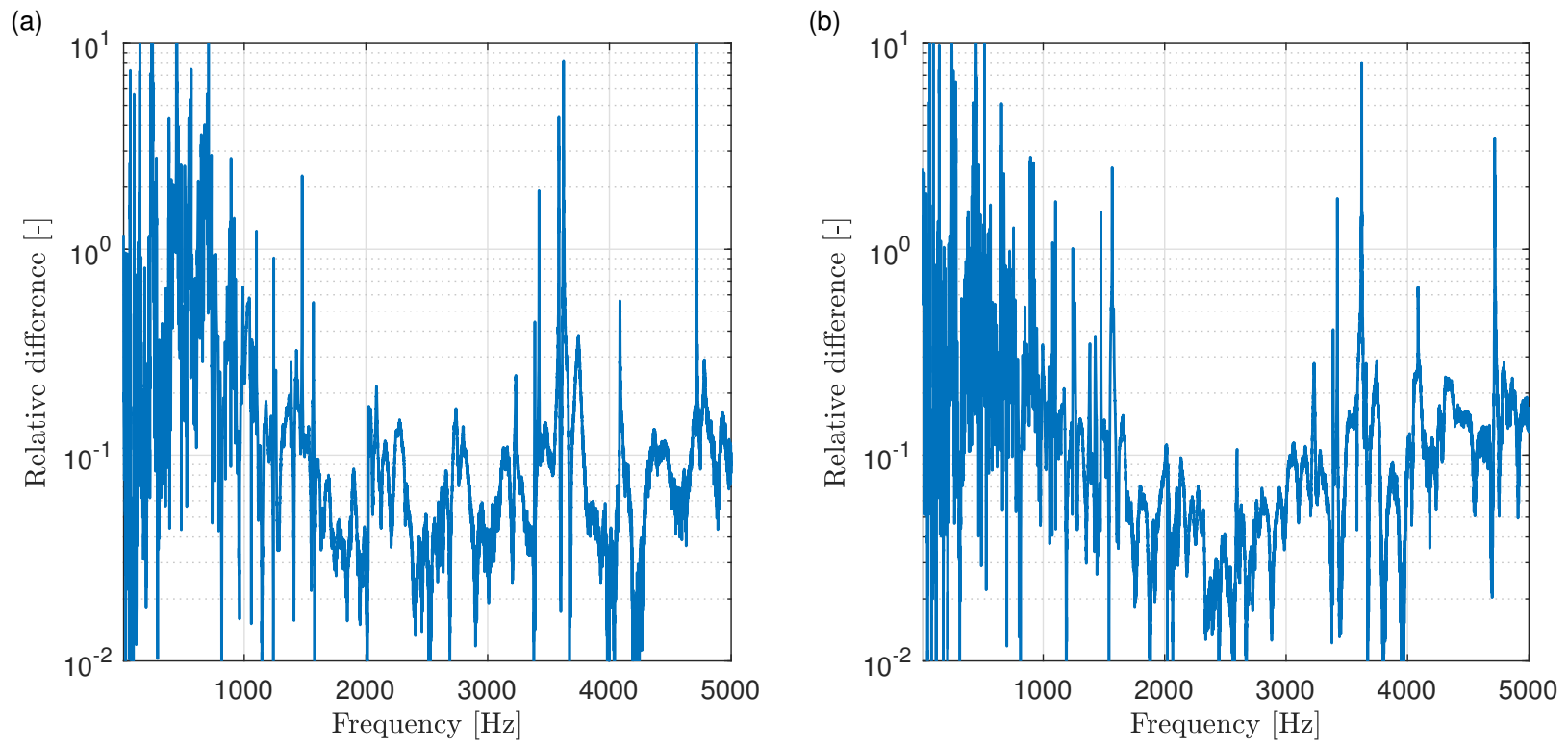

Figure 5: Relative difference in the $\mathrm{AE}$ condition for the case of a plate with added damping and $f(\mathbf{A})=\mathbf{A}^{-1}$. (a) Relative difference $\left|\left(\mathrm{E}\left[\mathbf{A}^{-1}\right]-\mathrm{E}[\mathbf{A}]^{-1}\right)_{11}\right| /\left|\left(\mathrm{E}\left[\mathbf{A}^{-1}\right]\right)_{11}\right|$. (b) Relative difference $\left|\left(\mathrm{E}\left[\mathbf{A}^{-1}\right]-\mathrm{E}[\mathbf{A}]^{-1}\right)_{12}\right| /\left|\left(\mathrm{E}\left[\mathbf{A}^{-1}\right]\right)_{12}\right|$

The AE condition is expected to be satisfied by any function $f(\quad)$ that has a convergent Taylor series expansion. Figure 6 tests the validity of the AE condition for $f(\mathbf{A})=\mathbf{A}^{2}$ using the ensemble of accelerance matrices obtained from the un-damped plate. As before, the variability over the ensemble is also included by plotting $\mathbf{A}^{2}$ for each member of the experimental ensemble. The results show that the AE is clearly satisfied across almost the entire range of frequencies considered. Discrepancies are again only observed at low frequencies and, as before, this can be justified by the fact that the amount of randomness added to the structure is insufficient to ensure that the AE conditions are satisfied. 

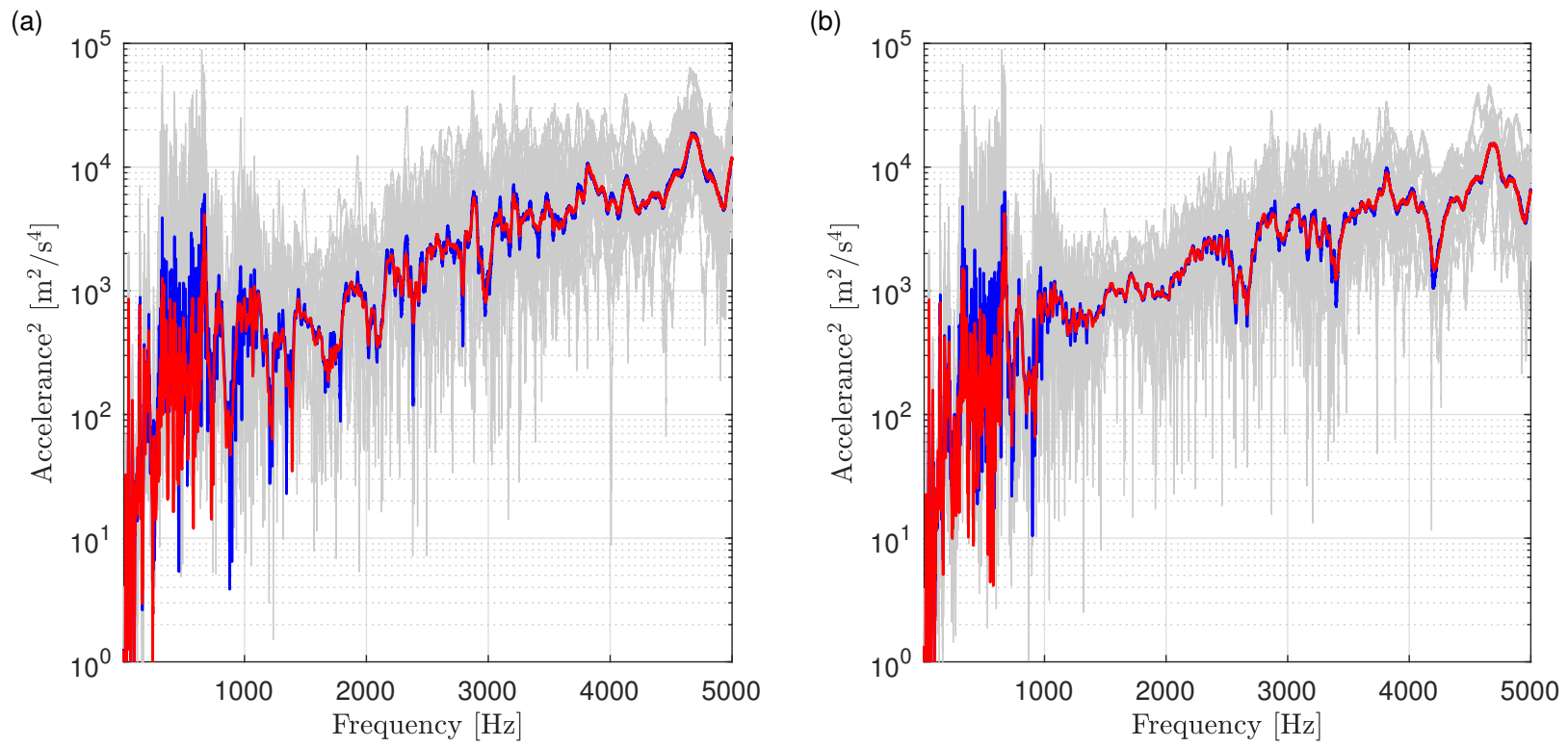

Figure 6: Experimental verification of the $\mathrm{AE}$ condition using the accelerance matrix of the plate without added damping, and with $f(\mathbf{A})=\mathbf{A}^{2}$. (a) Modulus of $\mathrm{E}\left[\left(\mathbf{A}^{2}\right)_{11}\right]$ (blue), $\left(\mathrm{E}[\mathbf{A}]^{2}\right)_{11}$ (red) and $\left(\mathbf{A}^{2}\right)_{11}$ (gray). (b) Modulus of $\mathrm{E}\left[\left(\mathbf{A}^{2}\right)_{12}\right]($ blue), $\left(\mathrm{E}[\mathbf{A}]^{2}\right)_{12}$ (red) and $\left(\mathbf{A}^{2}\right)_{12}$ (gray).

This subsection has presented an experimental verification of the AE condition for random causal FRFs. An application of this result will be shown in the next subsection, where the experimental determination of a direct field dynamic stiffness is investigated.

\subsection{An experimental direct field dynamic stiffness}

The hybrid FE-SEA method presented by Shorter and Langley $[3]$ considers a complex structure as an assembly of components with a dynamic response that is either highly sensitive or insensitive to random manufacturing uncertainties. These are identified collectively as the statistical subsystems, and the FE/deterministic system, respectively. The method assumes that the statistical subsystems are either connected to deterministic components, or to other statistical components, by deterministic junctions. With this assumptions, the response of all the deterministic components is represented by a set of displacement degrees of freedom (dof) $\mathbf{q}_{\mathrm{d}}$, and the response of each statistical subsystem is represented by its (ensemble and time average) vibrational energy $E$. Following the formulation presented in [5], a subset of these displacements are referred to as the boundary dofs q. It is through these boundary dofs that a statistical subsystem is connected to either the deterministic system, or other statistical subsystems. Then, for a given harmonic frequency $\omega$, the governing equations of motion if external forces $\mathbf{f}(\omega)$ are applied to the boundary dofs are

$$
\mathbf{D}(\omega) \mathbf{q}(\omega)=\mathbf{f}(\omega),
$$


where $\mathbf{D}(\omega)$ is the dynamic stiffness matrix of the subsystem, and $\mathbf{f}(\omega)$ is the force applied at the boundary dof. The $\omega$ dependence will be later omitted for brevity.

The hybrid FE-SEA method considers that the wave field generated in a statistical subsystems can be understood as the combination of the initially generated waves (direct field), and the waves generated by the reflections at the subsystem's unknown boundaries (reverberant field). The direct field contribution is represented by a direct field dynamic stiffness matrix $\mathbf{D}_{\text {dir }}$, which can be understood as the dynamic stiffness contribution of the statistical subsystem, at the boundary dofs $\mathbf{q}$, if there were no unknown boundaries in the subsystem [3, i.e. if the subsystem was infinite and waves emanating from $\mathbf{q}$ were not reflected back to it. The added contribution of the reverberant field is included by means of a blocked reverberant force $\mathbf{f}_{\text {rev }}$. By definition, the direct field contribution is equal for all the members of an ensemble of random systems, but the reverberant field contribution varies along the ensemble. Taking all this into account, Eq. (1) can be then expressed as

$$
\mathbf{D}_{\mathrm{dir}} \mathbf{q}=\mathbf{f}+\mathbf{f}_{\mathrm{rev}}
$$

where the dynamic stiffness $\mathbf{D}$ contribution has been separated into the (deterministic) direct field dynamic stiffness $\mathbf{D}_{\text {dir }}$ and the (random) reverberant field force vector $\mathbf{f}_{\mathrm{rev}}$. If the considered statistical subsystem carries a diffuse field over the ensemble [4, then $E\left[\mathbf{f}_{\text {rev }}\right]=0$ and, taking the ensemble average in Eq. (2) and considering that the applied force is deterministic, the expected value of the system response can be written as

$$
\mathrm{E}[\mathbf{q}]=\mathbf{D}_{\text {dir }}^{-1} \mathbf{f}
$$

On the other hand, by inverting Eq. (1) and taking the ensemble average, this response is given by

$$
\mathrm{E}[\mathbf{q}]=\mathrm{E}[\mathbf{H}] \mathbf{f}
$$

where $\mathbf{H}=\mathbf{D}^{-1}$ is the receptance matrix of the system. Then, considering the AE condition for the case $f(\mathbf{H})=\mathbf{H}^{-1}$, which has been discussed in the previous subsection, the direct field dynamic stiffness can be finally expressed as

$$
\mathbf{D}_{\text {dir }}=\mathrm{E}[\mathbf{H}]^{-1}=\mathrm{E}[\mathbf{D}]
$$

Eq. (5) was previously deduced in [5] without explicitly invoking the AE condition.

Several analytical and numerical techniques have been proposed to evaluate the $\mathbf{D}_{\text {dir }}$. Examples for point, line and area junctions can be found in [13, 6] and [14, respectively. Eq. (5), however, suggests an alternative method for determining it: Provided that (i) the receptance matrix $\mathbf{H}$ associated to the 
boundary dof can be measured, and (ii) an ensemble of random subsystems can be built experimentally, $\mathbf{D}_{\text {dir }}$ can be determined as an ensemble average of measured dynamic stiffness. The validity and advantages of the proposed approach are presented in the following subsections, which use the sets of experimental data described in Section 2 to discuss three types of point connections in a thin plate.

\subsubsection{Interior single point connection}

The direct field dynamic stiffness of a point connection far from any of the plate's edges can be obtained using a wave analysis [13. In general six physical dof are associated to a point connection and the corresponding $\mathbf{D}_{\text {dir }}$ will be a $6 \times 6$ matrix. However, if the component connected to the statistical thin plate is a resilient element, such as a antivibration mount, it may be sufficient to consider the dof that represents the displacement perpendicular to the plate, referred here as vertical displacement. Then, $\mathbf{D}_{\text {dir }}$ can be computed using the vertical driving-point response of an infinite thin plate $H_{\mathrm{dp}}$. This stiffness can be expressed as [19]

$$
D_{\mathrm{dir}}=H_{\mathrm{dp}}^{-1}=8 \mathrm{i} \omega \sqrt{D_{p} \rho h}
$$

where $\rho$ is the plate's mass density, $h$ is its thickness, and $D_{p}=E h^{3} / 12\left(1-\nu^{2}\right)$ is its flexural rigidity, $E$ being its Young's modulus and $\nu$ its Poisson's ratio.

According to Eq. (5), $\mathbf{D}_{\text {dir }}$ can be also obtained using the experimental results described in Section 2 In this case, the dynamic stiffness can be expressed as

$$
D_{\text {dir }}=\mathrm{E}[D]=-\omega^{2} \mathrm{E}\left[A_{i i}^{-1}\right]=-\omega^{2}\left(\mathrm{E}\left[A_{i i}\right]\right)^{-1}
$$

where $A_{i i}$ is a diagonal component of the measured accelerance matrix related to a point in the interior of the plate, and where the $\mathrm{AE}$ condition has been used in the rightmost equality.

Figure 7 compares the (a) real and (b) imaginary components of the analytical dynamic stiffness obtained from Eq. (6), with the experimental dynamic stiffness obtained using Eq. (7). The dynamic stiffness $D$ corresponding to each member of the experimental ensemble has been also included in the figure. To take into account the presence of the accelerometers, a mass correction factor has been applied to the experimental results 25 . The results correspond to the first dof marked in Figure 1a but very similar results where obtained for dof two and three. 

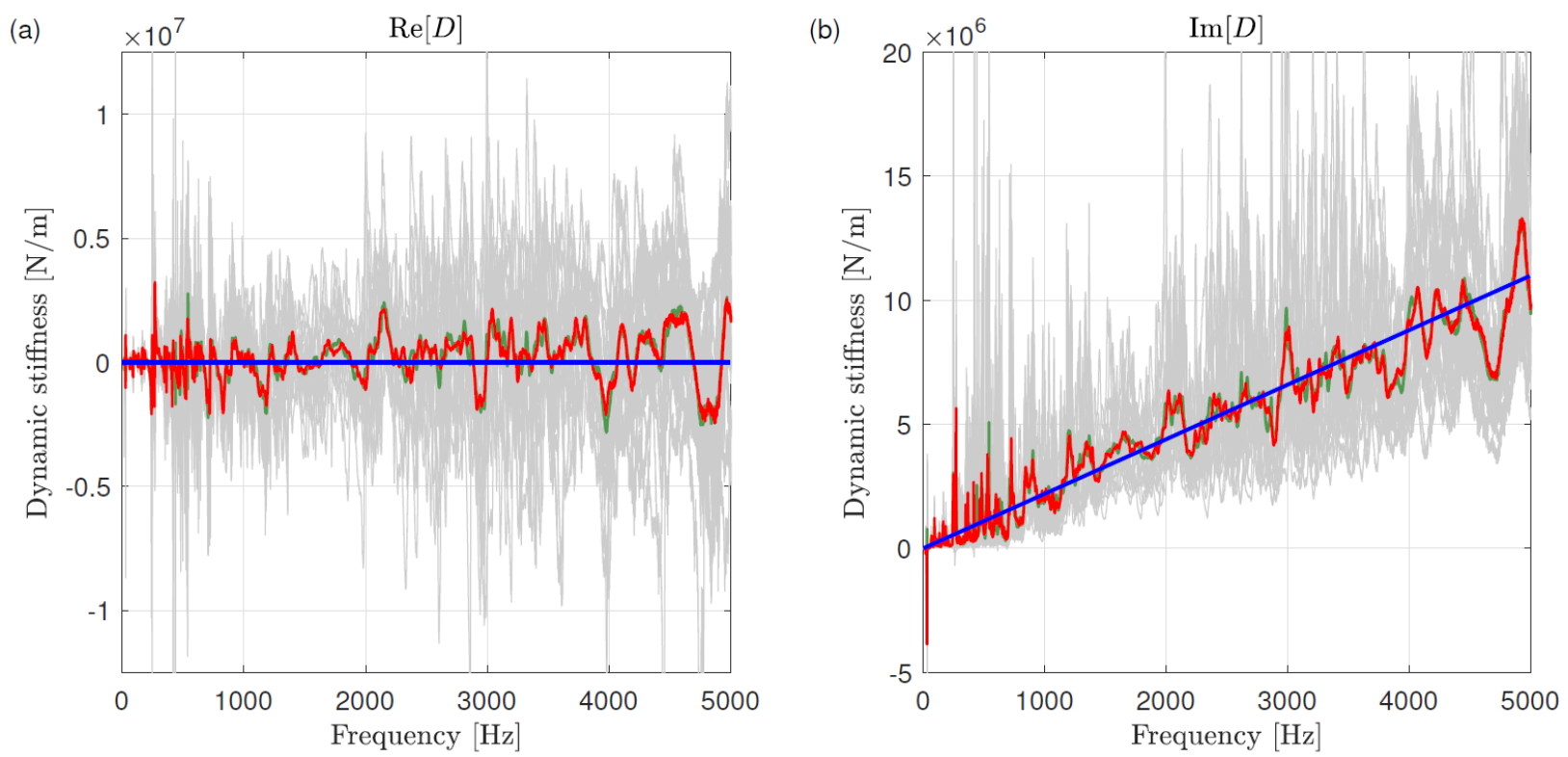

Figure 7: Direct field dynamic stiffness for a single point connection far from the plate's edges. Red: Experimental ensemble mean using $f(\mathrm{E}[H])$; Green: Experimental ensemble mean using $\mathrm{E}[f(H)]$; blue: analytical expression; gray: Dynamic stiffness of the 20 members of the ensemble. (a) Real components. (b) Imaginary components.

The results show a very good agreement between the ensemble average of the experimental dynamic stiffness and the analytical expression for $D_{\text {dir }}$. As expected the real component of the ensemble average dynamic stiffness oscillates around zero. Almost no difference can be observed between inverting the ensemble average receptance and ensemble averaging the dynamic stiffness. This result is consistent with the AE condition tests presented in Subsection 3.1 .

\subsubsection{Interior multi-point connection}

If one or more resilient elements are connected to the statistical thin plate via multiple point connections that are close to each other, then it may be necessary to take into account the coherence between them. If, as in the previous case, it is assumed that it is sufficient to consider only the vertical displacement of each point connection, then $\mathbf{D}_{\text {dir }}$ can be computed using the vertical response of an infinite plate to vertical excitations. Considering $i$ as the response position and $j$ as the position where the vertical point load is applied, the plate response can be expressed as [19]

$$
H_{i j}=H\left(r_{i j}\right)=\frac{H_{0}^{(2)}\left(k_{B} r_{i j}\right)-(2 \mathrm{i} / \pi) K_{0}\left(k_{B} r_{i j}\right)}{8 \mathrm{i} \omega \sqrt{D_{p} \rho h}},
$$

\footnotetext{
where $H_{0}^{(2)}$ is the zeroth order Hankel function of the second kind, $K_{0}$ is the zeroth order modified Bessel function of the second kind, $r_{i j}$ is the distance between both positions and $k_{B}=\left(\rho h \omega^{2} / D_{p}\right)^{1 / 4}$ is the plate bending wavenumber.
} 
Eq. (8) can be used to build a matrix of receptances $\mathbf{H}$ for an arbitrary number dofs representing vertical displacements far from any edge of the plate. Then $\mathbf{D}_{\text {dir }}$ can be obtained by inverting $\mathbf{H}$. In the case of a two-point connection, the dynamic stiffness can be written as follows

$$
\mathbf{D}_{\mathrm{dir}}=\mathbf{H}^{-1}=\left(\begin{array}{ll}
H_{11} & H_{12} \\
H_{21} & H_{22}
\end{array}\right)^{-1}
$$

where $H_{11}=H_{22}$ and $H_{12}=H_{21}$ are computed using Eq. (8).

As before, Eq. (5) and the experimental results described in Section 2 can be also used to obtain the $\mathbf{D}_{\text {dir }}$ of a connection consisting of two interior points. In this case, the dynamic stiffness can be expressed as

$$
\mathbf{D}_{\text {dir }}=\mathrm{E}[\mathbf{D}]=-\omega^{2} \mathrm{E}\left[\mathbf{A}^{-1}\right]=-\omega^{2} \mathrm{E}[\mathbf{A}]^{-1}
$$

where, A now represents a $2 \times 2$ block of the measured $6 \times 6$ accelerance matrix, and again, the AE condition has been used in the rightmost equality.

Figure 8 compares the $(\mathrm{a}, \mathrm{c})$ real and $(\mathrm{b}, \mathrm{d})$ imaginary components of the driving $(\mathrm{a}, \mathrm{b})$ and transfer $(\mathrm{c}, \mathrm{d})$ components of $\mathbf{D}_{\text {dir. }}$. The analytical dynamic stiffness matrix is obtained from Eq. (9) and the experimental dynamic stiffness is obtained using Eq. 10p. The dynamic stiffness $\mathbf{D}$ corresponding to each member of the experimental ensemble has been also included in the figure. The positions considered in this case are dofs one and three in Figure 1a, which are $11 \mathrm{~cm}$ apart. Again, the presence of accelerometers have been taken into account by applying a mass correction to the experimental results [25]. 

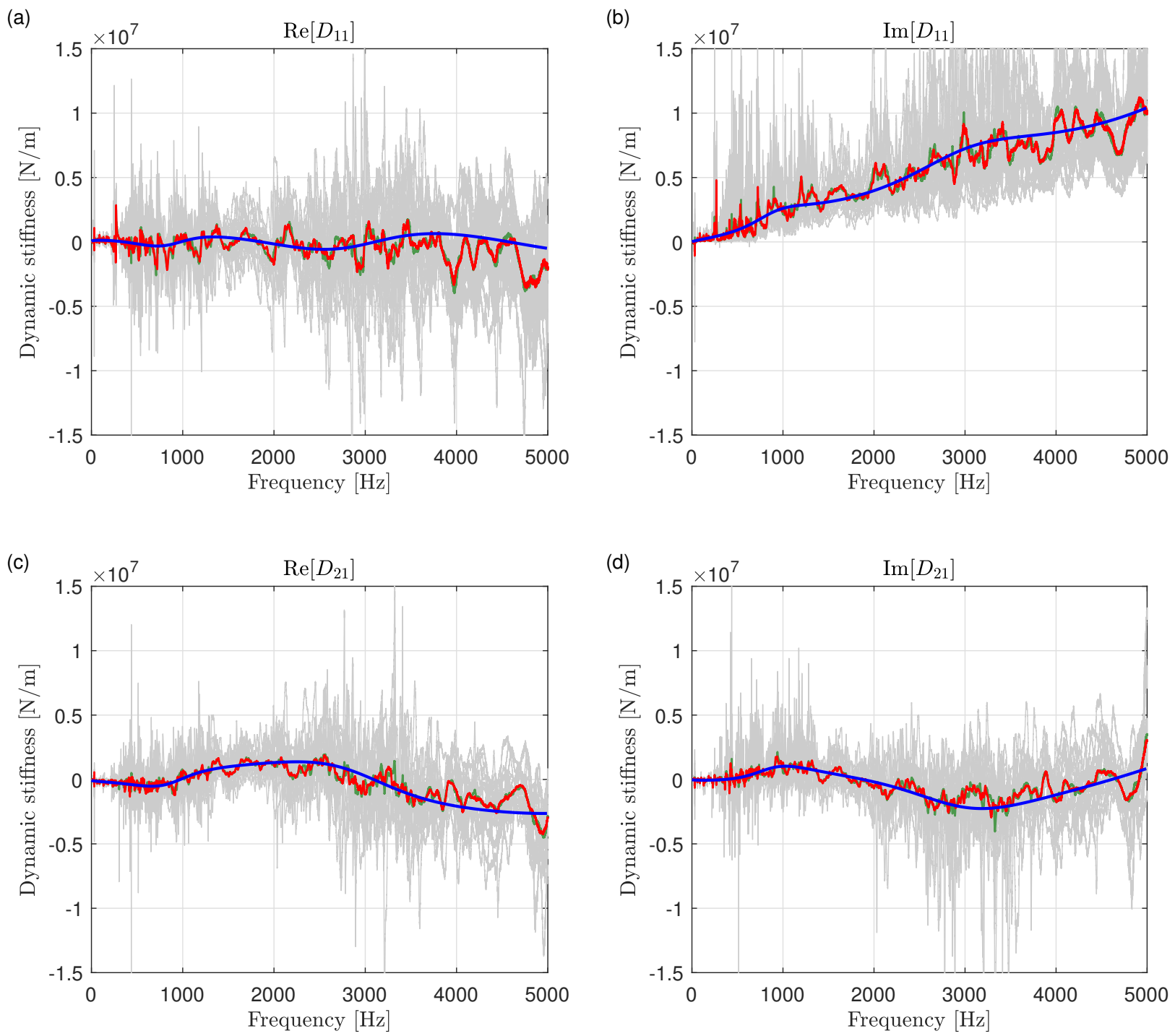

Figure 8: Direct field dynamic stiffness for a two-point connection far from the plate's edges. Red: Experimental ensemble mean using $\mathbf{f}(\mathrm{E}[\mathbf{H}])$; Green: Experimental ensemble mean using $\mathrm{E}[\mathbf{f}(\mathbf{H})]$; blue: analytical expression; gray: Dynamic stiffness of the 20 members of the ensemble. (a) Real part of a driving component. (b) Imaginary part of a driving component. (c) Real part of a transfer component. (d) Imaginary part of a transfer component.

The results show again a very good agreement between the ensemble average of the experimental dynamic stiffness and the analytical expression for $D_{\text {dir }}$. This agreement can be seen in both driving and transfer components of the stiffness. As before, the agreement found between the result of inverting the ensemble average receptance and of ensemble averaging the dynamic stiffness shows that the AE condition is clearly satisfied. 


\subsubsection{Near-edge point connection}

The cases of a single and a multiple point connection presented in the previous subsections have shown that experimental data can be used to obtain the $\mathbf{D}_{\text {dir }}$ associated to a deterministic junction. In these two cases, however, the advantage of using this method is rather limited, as rather simple analytical expressions can be obtained. The case presented in this subsection, that of a point lying near one of the edges of the plate, offers a first insight of the potential of the proposed method.

In general, the previously presented analytical expressions for points in the interior of a plate cannot be used for a point that lies near one of its edges. The edge may have a deterministic effect on the ensemble response that has to be included in $\mathbf{D}_{\text {dir }}$. This work presents a numerical strategy to compute the direct field dynamic stiffness for such cases. The method assumes that the point is near the edge of a semi-infinite plate, i.e. assumes that other edges of the plate are part of the unknown boundaries of the statistical system. The dynamic stiffness of interest is then obtained combining the dynamic stiffness of a plate strip with a width equal to the point-edge distance, and the dynamic stiffness of the edge of a semi-infinite plate. The details of the formulation can be found in Appendix A As before, an experimental $\mathbf{D}_{\text {dir }}$ can be obtained using Eq. (7) from the experimental results described in Section 2

Figure 9 compares the (a) real and (b) imaginary components of the analytical dynamic stiffness obtained using the numerical approach described in Appendix A, with the experimental dynamic stiffness obtained using Eq. (7). The dynamic stiffness $D$ corresponding to each member of the experimental ensemble has been also included in the figure. In this case, the results correspond to the sixth dof marked in Figure 1 a and, as before, a correction factor was applied to take into account the accelerometer mass. 

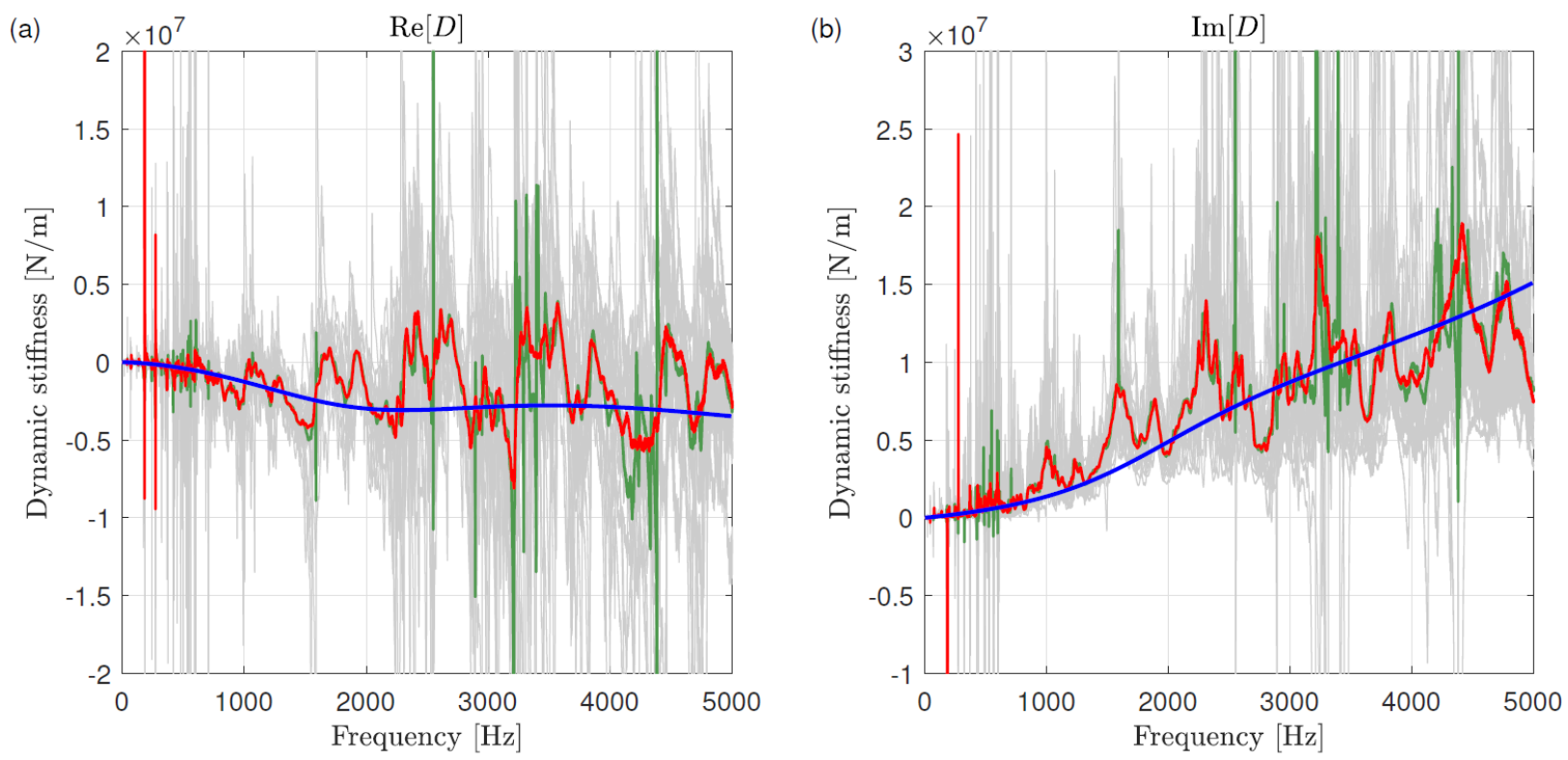

Figure 9: Direct field dynamic stiffness for a single point connection near one plate edge. Red: Experimental ensemble mean using $f(\mathrm{E}[H])$; Green: Experimental ensemble mean using $\mathrm{E}[f(H)]$; blue: analytical expression; gray: Dynamic stiffness of the 20 members of the ensemble. (a) Real components. (b) Imaginary components.

The results show a good agreement between the ensemble average of the experimental dynamic stiffness and the analytical expression for $D_{\mathrm{dir}}$. In this case, the result of inverting the ensemble average receptance is clearer than the result of ensemble averaging the dynamic stiffness. In such situations, the AE condition can be invoked, and the better result can be used.

The results presented in this last subsection show that the experimental approach is suitable for modelling connections that may be significantly challenging to represent using analytic or numerical techniques. However, in some cases the procedure to obtain an ensemble of "nominally identical" subsystems, by randomising experimentally the original structure as described in Section 2, may not be applicable. An alternative procedure, which combines the use of experimental measurements and analytical results, is presented in the next section.

\section{Extending the ensemble using virtual masses}

The results shown in Section 3 suggest that experimental measurements can be used to determine $\mathbf{D}_{\text {dir }}$ for those connections that can be challenging (or even impossible) to represent using analytical or numerical techniques. As it has been explained in Section 2, an ensemble of random subsystems can be built experimentally by attaching point masses at random locations of a structure. However, there may be cases in which either the nature of the structure impedes the attachment of these additional masses on it, or the procedure becomes extremely time consuming. For these cases, an alternative method is proposed in this 
section. The method is based on the generation of new members of an ensemble of "nominally identical" systems without having to physically randomise it. Therefore, to differentiate them from the ensembles obtained using the method described in Section 2 , ensembles that have been created using this new approach will be referred as "artificial ensembles".

\subsection{Description of the proposed methodology}

The aim of the proposed methodology is to reproduce the procedure described in Section 2 adding the random point masses numerically instead of physically attaching them. The procedure consist then of the following steps:

1. A set of measurement positions are defined. This set consists of:

- $N_{I}$ positions of interest. For the rectangular plate discussed in Section 2 these would be the six points marked with red dots in Figure 1a

- $N_{P}$ additional positions where "artificial" point masses will be added. These positions should be randomly distributed along the system and, as it will be later discussed, $N_{P}$ should, in principle, be significantly larger than the number of masses $N_{M}$ that will be numerically added.

2. Impact excitations are applied at each measurement position using an instrumented hammer, and the response at all positions is measured using accelerometers. For those positions (or dofs) that cannot be directly accessed or excited, alternative techniques such as the round trip method [26] can be considered.

3. An initial accelerance matrix $\mathbf{A}_{\text {ini }}$ is obtained dividing the measured acceleration spectra by the measured force spectrum for each excitation. Note that this accelerance matrix will be significantly larger than the one obtained in Section 2. It is however, only measured once.

4. The corresponding initial apparent mass matrix is obtained by inversion as $\mathbf{M}_{\text {ini }}=\mathbf{A}_{\text {ini }}^{-1}$.

5. For each member $i$ of the ensemble, a modified apparent mass matrix is obtained,

$$
\mathbf{M}_{\mathrm{mod}, \mathrm{i}}=\mathbf{M}_{\mathrm{ini}}+\mathbf{M}_{\mathrm{add}, \mathrm{i}}
$$

where $\mathbf{M}_{\mathrm{add}, \mathrm{i}}$ is a diagonal matrix that contains the apparent mass matrix contribution added by the $N_{M}$ numerical point masses. Therefore, the only non-zero components of this matrix are those $N_{M}$ diagonal components that correspond to the positions where the point masses have been added, and these will be equal to the added point mass $m_{\text {added }}$. For each ensemble member $i$ the positions of the point masses are chosen randomly from the larger set of positions $N_{P}$. It is important to note that (i) point masses should not be added to any of the $N_{I}$ positions of interest, and (ii) if $N_{P}$ is not significantly larger than $N_{M}$ there will be a limited number of possible mass "permutations", and a 
limited amount of uncertainty in the obtained artificial ensemble. The later limitation, however, can be overcome to some extent if the amount of mass $m_{\text {added }}$ added is also considered to be random over the ensemble. Note also that, with this method, alternative components, such as a random stiffness, could be also included with no extra effort.

6. For each member $i$ of the ensemble, the corresponding modified accelerance matrix is obtained inverting the modified apparent mass matrix $\mathbf{A}_{\text {mod,i }}=\mathbf{M}_{\text {mod,i }}^{-1}$. Each one of these modified accelerance matrices can be expressed as

$$
\mathbf{A}_{\text {mod }, \mathrm{i}}=\left(\begin{array}{ll}
\mathbf{A}_{d_{i} d_{i}, i} & \mathbf{A}_{d_{i} d_{p}, i} \\
\mathbf{A}_{d_{p} d_{i}, i} & \mathbf{A}_{d_{p} d_{p}, i}
\end{array}\right)
$$

where $d_{i}$ refers to the dofs that are not modified in the randomisation strategy, i.e. the positions of interest, and $d_{p}$ refers to those that are.

There are some potential benefits of building an artificial ensemble instead of an experimental one. The method for building them will be, in most cases, significantly less time consuming than the physical randomisation of the system, specially for those cases where a large ensemble may be required. The efficiency of the new methodology is particularly clear when $N_{I}$, and therefore the size of $\mathbf{A}_{\text {ini }}$, is rather small. As mentioned before, this small size could be achieved by considering that the mass values $m_{\text {added }}$ of each one of the added point masses is a random value. Finally, it should be also mentioned that the applicability of the proposed method is not limited to the experimental cases described in this work and, for example, the method could be also applied to randomise an FE model of a statistical system.

In the next subsection the proposed methodology has been used to obtain an artificial ensemble of plates using the rectangular aluminium plate described in the previous sections.

\subsection{Results obtained using artificial ensembles}

\subsubsection{Plate without added damping}

The proposed methodology has been used to build an artificial ensemble of thin rectangular plates. The response of the plate to hammer impacts was measured, using accelerometers, at 30 different positions. These positions, which have been marked with dots in Figure 10b include the six positions considered in the experimental ensembles of section 3 , i.e. the positions of interest, and 24 additional positions randomly scattered across the plate structure. The artificial ensemble has been obtained considering $N_{I}=9$ positions of interest: the six measuring positions considered in Section 2 and three additional positions far from the plate's edges. For each member of the ensemble, point masses of $70 \mathrm{~g}$ have been added at 11 positions that are randomly chosen from the $N_{P}=30-N_{I}=21$ positions. As in the experimental case, the proposed method has been used to build an ensemble of 20 members. 


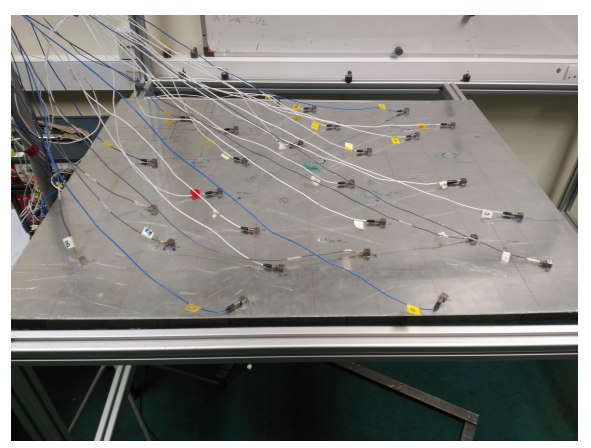

(a) Experimental set-up used for measuring the accelerance matrix $\mathbf{A}_{\text {ini }}$.

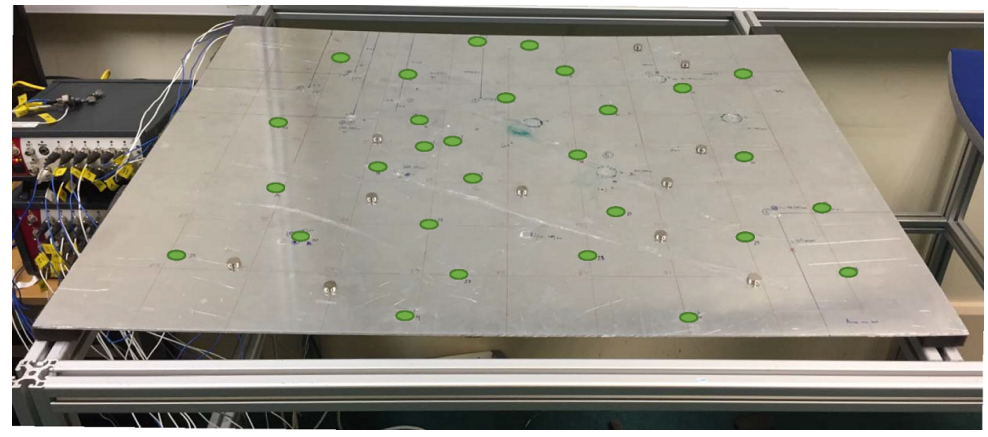

(b) Positions considered in measuring $\mathbf{A}_{\text {ini }}$ (green dots).

Figure 10: Experimental set-up for building an artificial ensemble of random plates

(a)

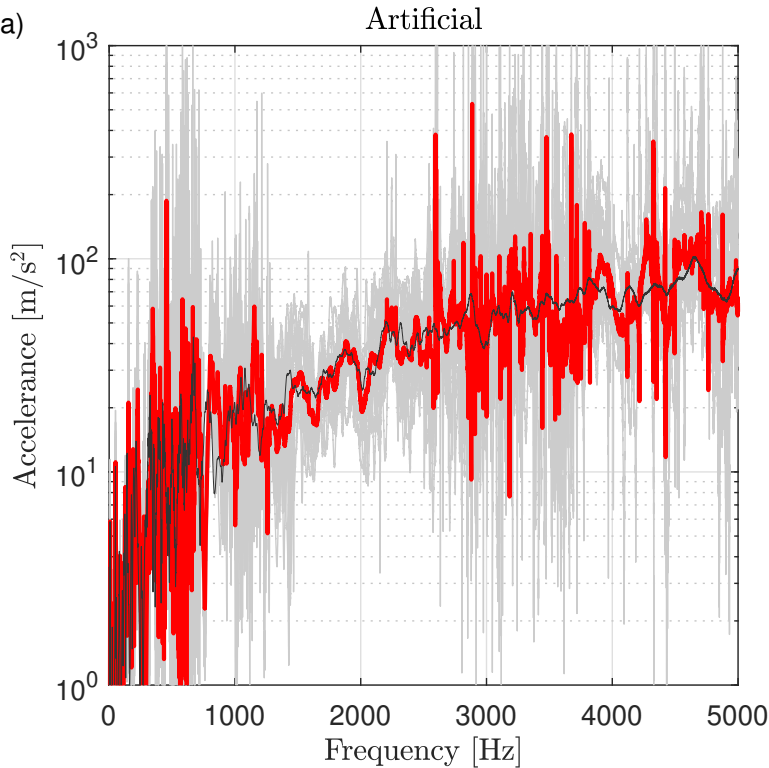

A comparison between the accelerance matrix obtained by physically randomising the system, and by virtual randomisation (i.e. using virtual point masses) is presented in Figure 11. The comparison shows the ensemble average of the driving component corresponding to the first dof (see Figure 1a). The accelerance for each of 20 ensemble members, both artificial and experimental, have also been included in the figure.

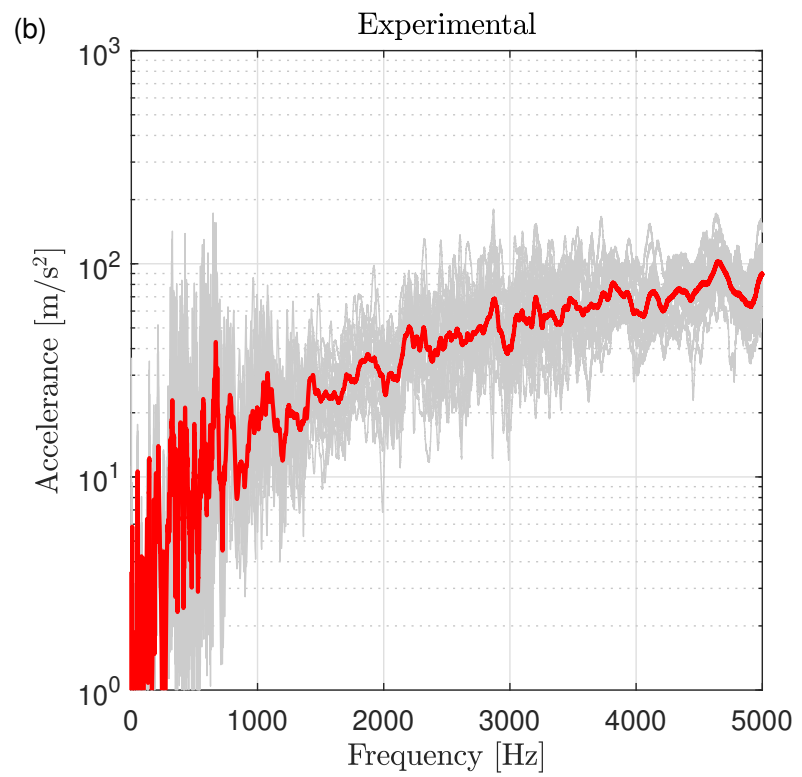

Figure 11: Accelerance matrix component $\mathbf{A}_{1,1}$. Red: Experimental ensemble mean; gray: Accelerance of the 20 members of the ensemble. (a) Artificial ensemble. The experimental ensemble mean has been included as a darker thin line (b) Experimental ensemble.

The results show that the accelerances obtained using virtual masses are considerably noisier than the experimental ones. This is clearly an unexpected result, as both methods are randomising the same dynamic structure. However, a direct comparison of accelerance components may be insufficient to quantify the im- 
portance of the differences observed, and further insight may be gained if the statistics of both experimental and artificial ensembles are compared to SEA mean and variance predictions. The expressions used to obtain these predictions are summarised in the following paragraphs.

The time and ensemble average vibrational energy $E$ of a single subsystem is given by 1

$$
\eta \omega E=P,
$$

where $\eta$ is the subsystem loss factor and $P$ is the time and ensemble average power input applied to the subsystem. For a point load excitation, it is shown in [1] that, when the resonance frequencies of the system are random and uniformly distributed over some frequency interval, the power input averaged over the source location is given by

$$
P=\frac{\pi n}{4 m_{\mathrm{p}}}|F|^{2},
$$

where $n$ is the subsystem's modal density (which, for the case of a plate, was defined in Section 2p, $m_{\mathrm{p}}$ is the mass of the statistical subsystem and $|F|$ is the amplitude of the excitation.

Langley and Brown 24] obtained an estimate of the response relative variance (i.e the variance divided by the square of the mean) by considering the statistics of the time averaged kinetic energy density of the system, which is given by

$$
T(\omega)=\sum_{n} \frac{\omega^{2} a_{n}}{\left[\left(\omega_{n}^{2}-\omega^{2}\right)^{2}+\left(\eta \omega \omega_{n}\right)^{2}\right]},
$$

where $\omega_{n}$ is the $n$th natural frequency of the system and $a_{n}$ are coefficients that depend on the type of loading assumed. In their study, by assuming that the natural frequencies of a subsystem are random and conform to the GOE statistics [27, 28, they obtained the following expression for the relative variance of a subsystem in terms of the modal overlap factor $m=\omega n \eta$ and of the spatial factor $\alpha=\mathrm{E}\left[a_{n}^{2}\right] / \mathrm{E}\left[a_{n}\right]^{2}$

$$
r^{2}(\alpha, m)=\frac{1}{\pi m}\left\{\alpha-1+\frac{1-\exp (-2 \pi m)}{2 \pi m}+E_{1}(\pi m)\left(\cosh (\pi m)-\frac{\sinh (\pi m)}{\pi m}\right)\right\},
$$

where $E_{1}$ is the exponential integral. The spatial factor for a single point load excitation is $\alpha=K=$ $\mathrm{E}\left[\phi_{n}^{4}(x)\right] / \mathrm{E}\left[\phi_{n}^{2}(x)\right]^{2}, \phi_{n}(x)$ being the mode shape at some location $x$. Numerical studies have shown that $K=2.75$ is an appropriate value for a plate structure [28, 24].

In the case of the experimental ensemble, the vibration energy of the plate has been estimated from the experimental results by averaging the response of two of the interior dof when the third one is excited to give $\left\langle|v|^{2}\right\rangle_{\mathrm{a}}$, and then noting that $\mathrm{E}[E]=m_{\mathrm{p}}\left\langle|v|^{2}\right\rangle_{\mathrm{a}} / 2$. With this approach, an ensemble of 20 experimental estimations of the plate energy have been obtained.

Figure 12 presents a comparison between the experimental vibrational energy of the plate and the energy predicted by the SEA equations. The ensemble mean and relative variance predictions have been calculated 
Figure 12: (a) Energy of the plate without added damping due to a unit point force excitation. Gray: response of the 20 members of the experimental ensemble; red: experimental ensemble mean response; blue: SEA prediction. (b) Relative variance of the energy. Red: experimental ensemble variance; blue: SEA prediction.

using Eqs. (13) and (16), respectively. The SEA predictions have been calculated using the mechanical parameters and loss factor defined in Section 2. The energy predicted for each one of the 20 members of the ensemble has been also included in the results.

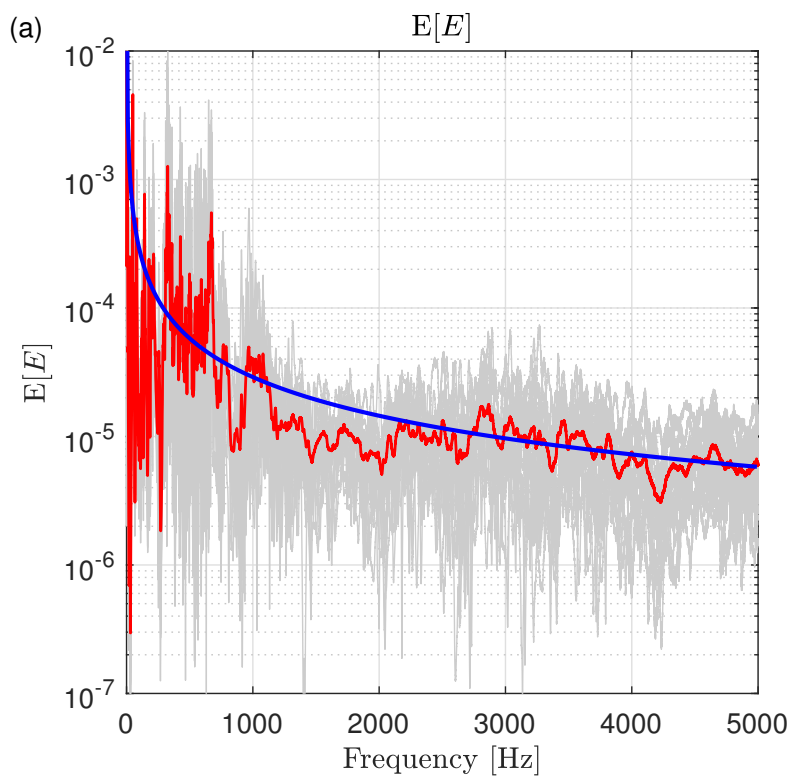

(a) (b)

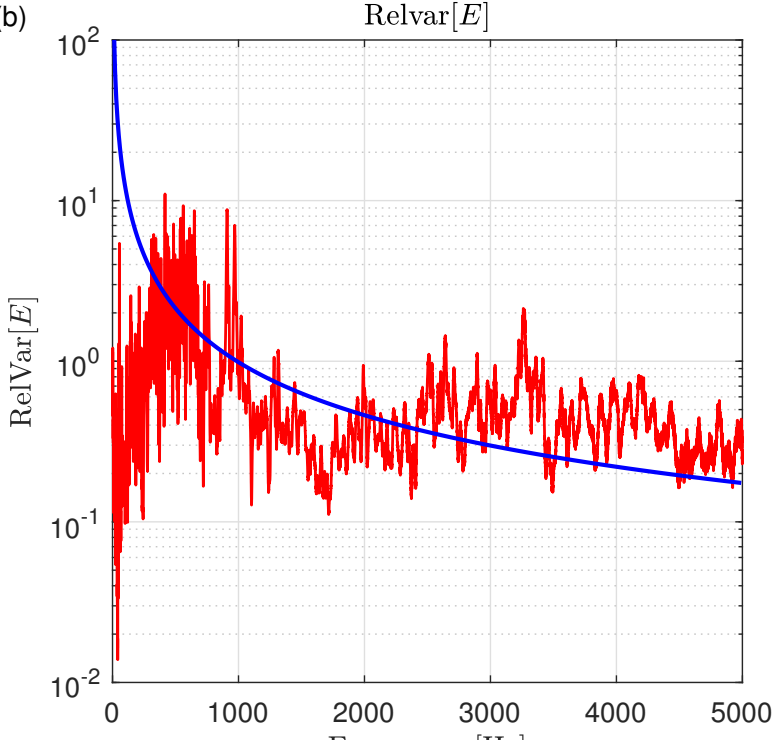

The results show a good agreement between the measured plate ensemble average response and the response predicted by the SEA equations. The SEA equations seem to slightly overestimate the response between $800 \mathrm{~Hz}$ and $2 \mathrm{kHz}$ but this can be explained by the frequency-dependent effect that the damping treatment has on the plate response. This effect can be also observed in the relative variance comparison. In both cases a slightly better agreement could be obtained if a frequency-dependent subsystem loss factor was used. Additional differences may arise as a result of the performed space averaging, which considers only two (near) positions. Nevertheless, the results show that the statistics of the experimental ensemble agree well with the theoretical predictions.

A similar comparison can be performed by estimating the vibration energy of the plate from the artificial ensemble of accelerances. It was mentioned before that, in obtaining of the artificial ensemble, $N_{I}=9$ positions of interest have been considered, and six of them are positions far from any of the plate's edges. In this case the vibration energy of the plate has been estimated by averaging the response of five of these interior dofs when the sixth one is excited.

Figure 13 presents a comparison between the vibrational energy of the plate estimated using the artificial ensemble results and the energy predicted by the SEA equations. As before, the SEA predictions have been 


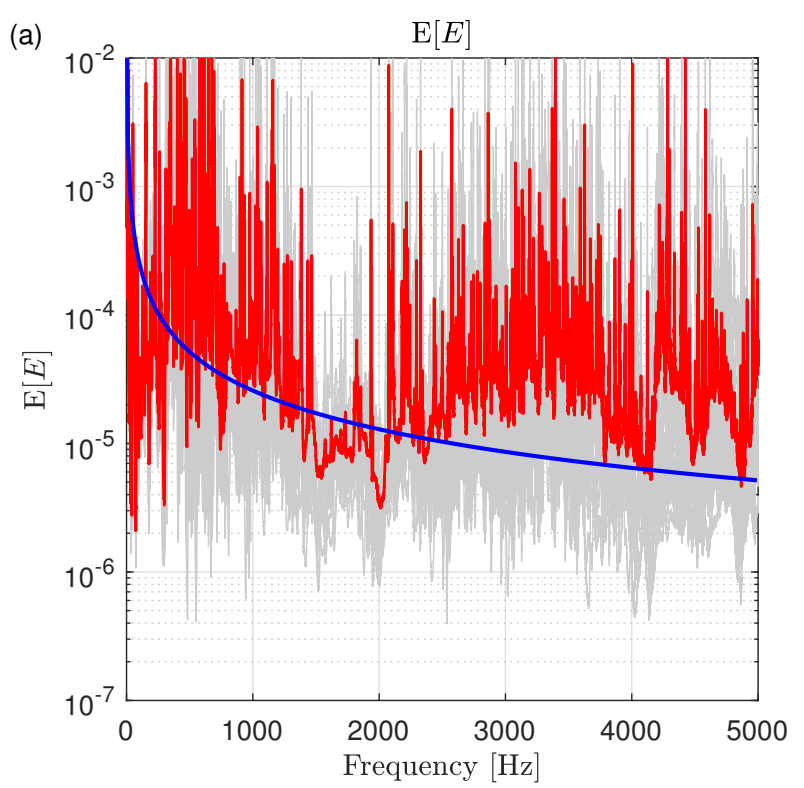
results. (b)

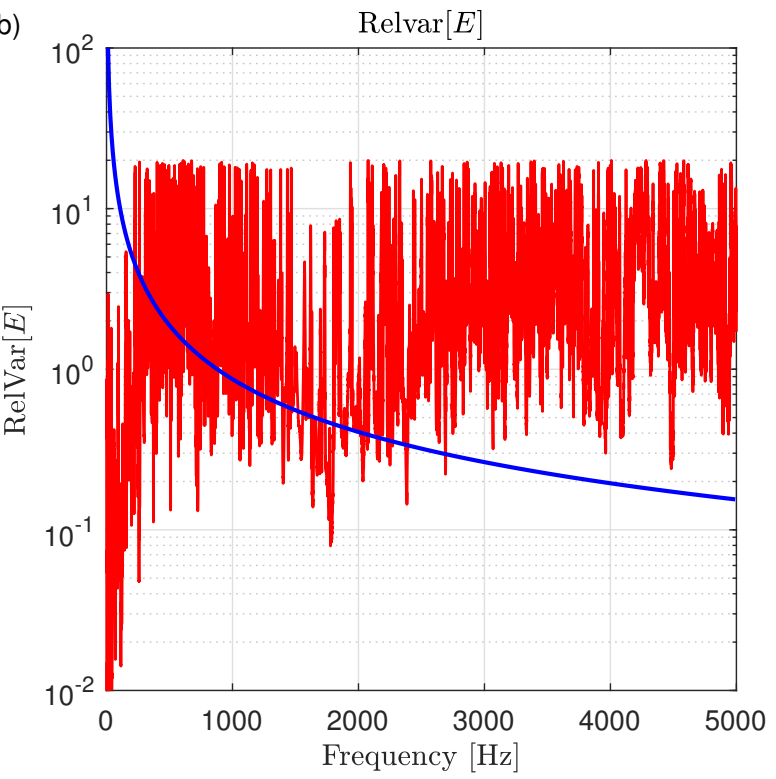

Figure 13: (a) Energy of the plate without added damping due to a unit point force excitation. Gray: response of the 20

members of the artificial ensemble; red: artificial ensemble mean response; blue: SEA prediction. (b) Relative variance of the energy. Red: artificial ensemble variance; blue: SEA prediction. calculated using Eqs. (13) and (16) with the mechanical parameters and loss factor defined in Section 2 Again, the energy predicted for each one of the 20 members of the ensemble has been also included in the

382

The results clearly show that both the ensemble mean and relative variance obtained from the artificial ensemble are extremely noisy. This fact suggests that the proposed methodology may have numerical or experis experimental issues that need to be understood. Further insights into the nature of these issues can be gained exining the components of the measured initial accelerance matrix $\mathbf{A}_{\text {ini }}$ and of the corresponding initial apparent mass of the system $\mathbf{M}_{\text {ini }}=\mathbf{A}_{\text {ini }}^{-1}$. The modulus of a component of each one of these $30 \times 30$ matrices shown in Figure 14 

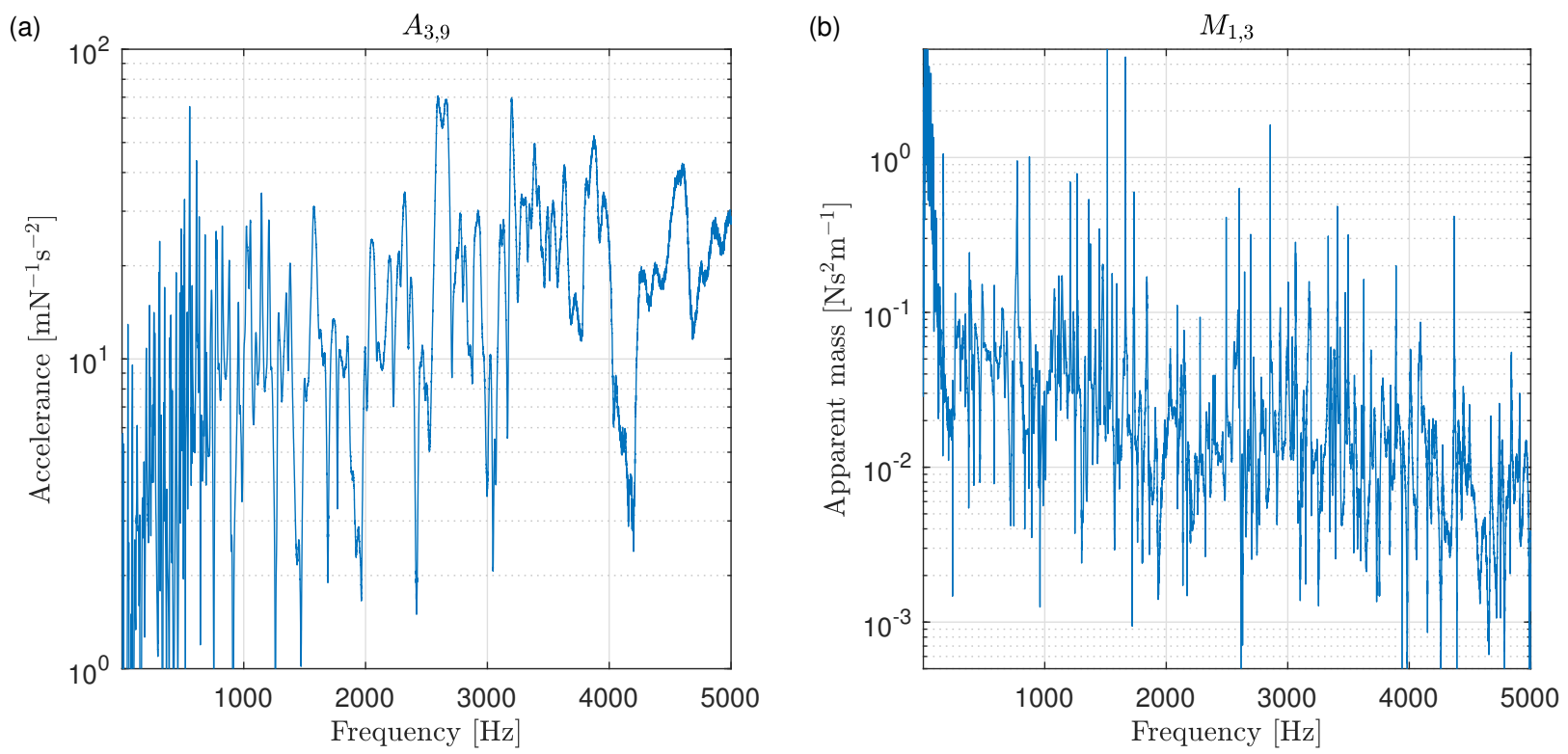

Figure 14: (a) Modulus of the component (3,9) of the initial accelerance matrix $\mathbf{A}_{\text {ini }}$ for the plate without added damping. (b) Modulus of the component (1,3) of the initial apparent mass matrix $\mathbf{M}_{\text {ini }}$ for the plate without added damping.

The frequency response of the accelerance component has an expected dynamic behaviour. There is an increase on the peaks bandwidth proportional to the excitation frequency, and at high frequencies the response is clearly smoothed due to the overlapping of modes. On the contrary, the results for the apparent mass component are rather unexpected. They exhibit a considerable number of very sharp peaks across the entire frequency range considered, and the bandwidth of these peaks seems to be insensitive to the corresponding excitation frequency. The correctness of the matrix inversion has been verified by checking that $\mathbf{M}_{\text {ini }} \mathbf{A}_{\text {ini }}-\mathbf{I}$ (where $\mathbf{I}$ is the identity matrix) is $\mathbf{0}$ to several significant digits. This result ensures that these peaks are not caused by a numerical instability of the matrix inversion, and that they may likely have an experimental cause.

Additional information can be obtained by noting that $\mathbf{M}_{\text {ini }}=\mathbf{A}_{\text {ini }}^{-1}=\operatorname{adj}\left(\mathbf{A}_{\text {ini }}\right) / \operatorname{det}\left(\mathbf{A}_{\text {ini }}\right)$, where adj stands for adjoint and det for determinant. Figure 15 presents an expanded view of one of these unexpected spikes in the apparent mass components (a), and details the frequency content of the modulus of $\operatorname{det}\left(\mathbf{A}_{\text {ini }}\right)$ and $\operatorname{adj}\left(\mathbf{A}_{\text {ini }}\right)$ around this spike. For the case of the determinant, the modulus of both real and imaginary components have been also included in the figure. 

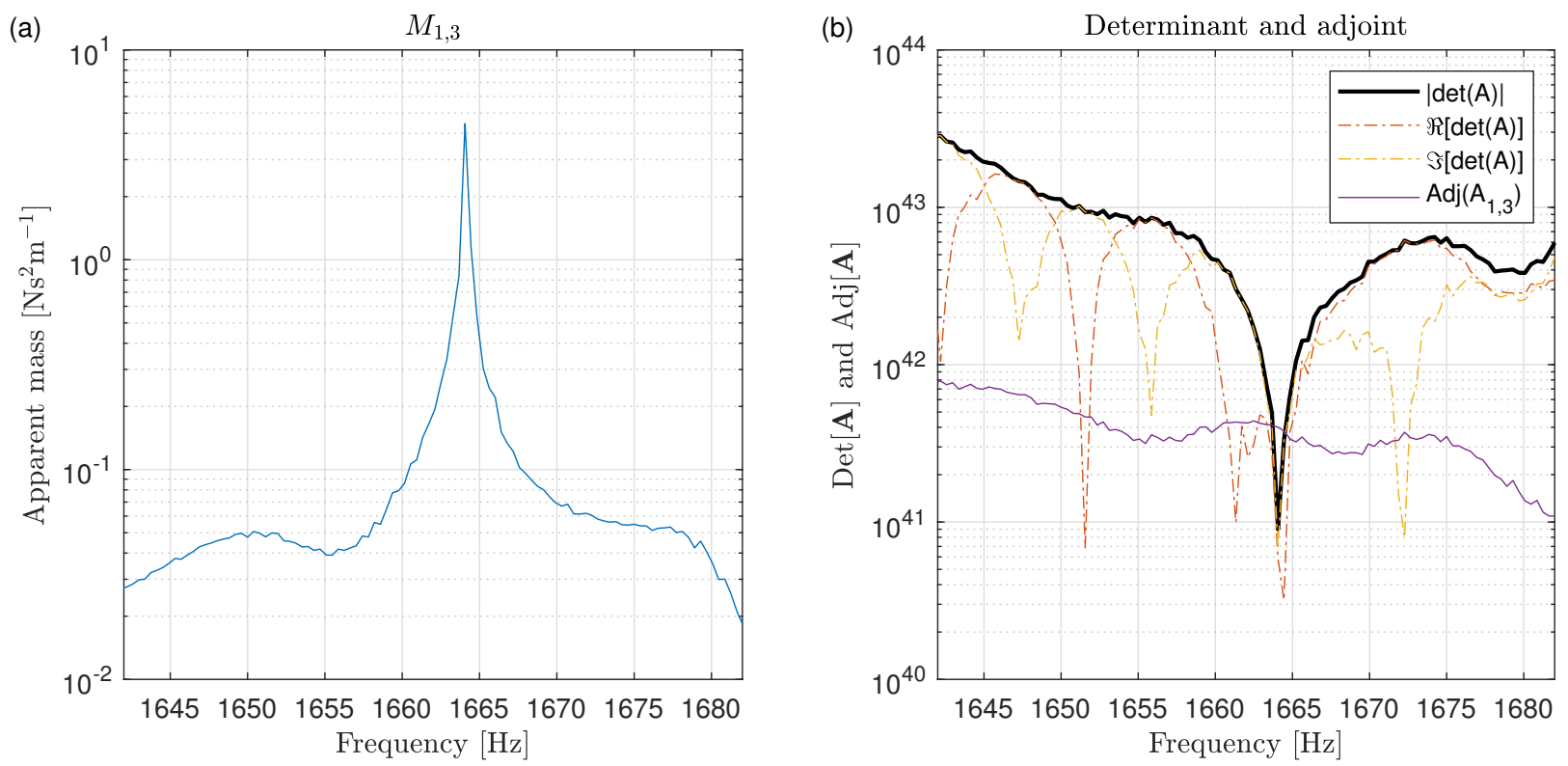

Figure 15: (a) Zoomed view of one of the spikes observed in the apparent mass matrix component shown in Figure 14 (b) Modulus of the terms involved in calculating $\mathbf{A}_{\text {ini }}^{-1}$. black: determinant; dashed blue: real component of the determinant; dashed red: imaginary part of the determinant, yellow: adjoint.

The results show that the spike occurrence is related to a sharp decay in the determinant value and not to a decay in the adjoint's magnitude. The same behaviour has been observed in many other apparent mass spikes.

The results presented in Figures 14 and 15 shows that main issues in building an artificial ensemble arise from inverting the experimentally determined initial accelerance matrix. As explained in Section 2 and Subsection 4.1, the dynamic response of the plate was obtained by applying impact excitations on each one of the accelerometers positions using an instrumented hammer. It is interesting to note that, due to the nature of the experimental procedure, the hammer impacts will have a limited precision, and may not be applied at the exact position where the corresponding accelerometer is located. In the next section, the effect that this imprecision on the obtained results is assessed using an analytical model of the plate.

\subsubsection{Exploration of the spikes}

The limited precision associated with the experimental determination of the accelerance matrix $\mathbf{A}_{\text {ini }}$ is studied in this section using an analytical model. The rectangular plate described in Section 2 is modelled here as a thin plate that is simply-supported on its four edges. The fact that the assumed boundary conditions do not represent the experimental ones is of little importance for the aim of this study. With these assumptions, the response $H\left(x_{r}, y_{r}\right)$ at a receiver position $\left(x_{r}, y_{r}\right)$ due to a harmonic unit point load 
excitation applied at a force position $\left(x_{f}, y_{f}\right)$ can be expressed as the following modal sum

$$
H\left(x_{r}, y_{r}\right)=\sum_{m=1}^{\infty} \sum_{n=1}^{\infty} \frac{\sin \left(\frac{n \pi x_{f}}{L_{x}}\right) \sin \left(\frac{m \pi y_{f}}{L_{y}}\right) \sin \left(\frac{n \pi x_{r}}{L_{x}}\right) \sin \left(\frac{m \pi y_{r}}{L_{y}}\right) \mathrm{e}^{-\mathrm{i} \phi_{n m}}}{C_{n m} \rho h \sqrt{\left(\omega_{n m}^{2}-\omega^{2}\right)^{2}+\omega_{n m}^{4} \eta^{2}}},
$$

where $L_{x}$ and $L_{y}$ are the length and width of the plate,

$$
\begin{gathered}
\phi_{n m}=\arctan \left(\frac{\eta}{1-\left(\omega / \omega_{n m}\right)^{2}}\right), \\
\omega_{n m}=\left[\left(\frac{n \pi}{L_{x}}\right)^{2}+\left(\frac{m \pi}{L_{y}}\right)^{2}\right] \sqrt{\frac{D_{p}}{\rho h}}
\end{gathered}
$$

and

$$
C_{n m}=\int_{0}^{L_{x}} \int_{0}^{L_{y}}\left[\sin \left(\frac{n \pi x}{L_{x}}\right) \sin \left(\frac{m \pi y}{L_{y}}\right)\right]^{2} \mathrm{~d} y \mathrm{~d} x=\frac{L_{x} L_{y}}{4} .
$$

Eq. 17) can be used to assess the effect of a potential experimental imprecision in the determination of the accelerance matrix $\mathbf{A}_{\text {ini }}$. This aim is achieved by building two FRF accelerance matrices. The first one considers that the force positions are collocated with the receiver positions $\left(x_{r_{1}}, y_{r_{1}}\right), \ldots,\left(x_{r_{N}}, y_{r_{N}}\right)$, which represents the (idealised) case where hammer impacts are applied exactly at each accelerometer position. The second case assumes a small random distance between excitation and response positions, so that $\left(x_{f_{k}}, y_{f_{k}}\right)=\left(x_{r_{k}}, y_{r_{k}}\right)+\left(\varepsilon_{x_{k}}, \varepsilon_{y_{k}}\right)$. This case represents the (realistic) situation where hammer impacts may not be exactly applied at the accelerometers positions. The obtained matrices are referred as collocated and non-collocated accelerance matrix, respectively.

The experimental procedure described in Subsection 4.1 has been simulated by choosing 30 positions across the plate. The positions have been placed randomly considering a minimum distance of $3 \mathrm{~cm}$ between them and the plate's edges. The random variables $\varepsilon_{x}$ and $\varepsilon_{y}$ are taken as uniformly distributed in the range $[-1,1] \mathrm{cm}$. The force and receiver positions considered for the case of the non-collocated accelerance matrix are shown in Figure 16. The mechanical properties and loss factor described in Section 2 have been used in the calculations. 


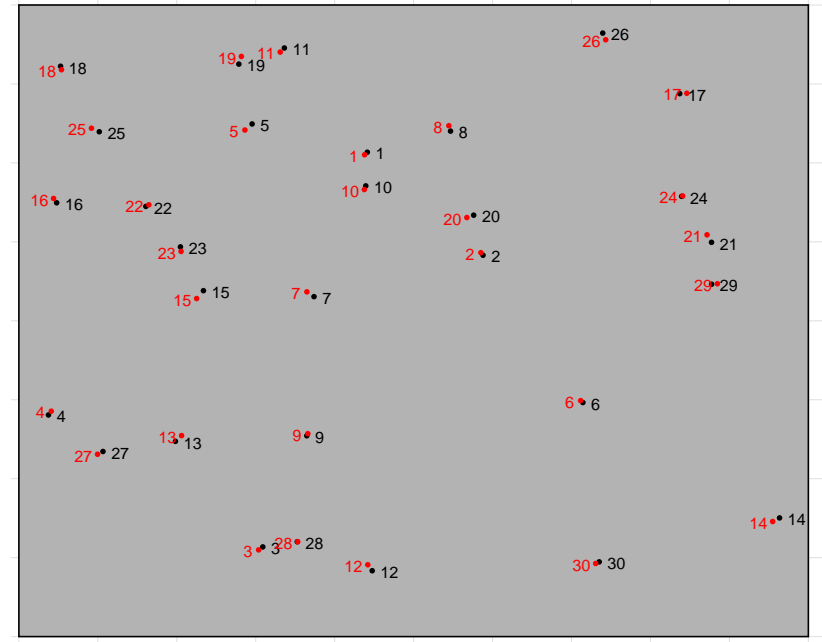

Figure 16: Force (red dots) and receiver (black dots) positions considered in the obtention of a non-collocated analytical accelerance matrix.

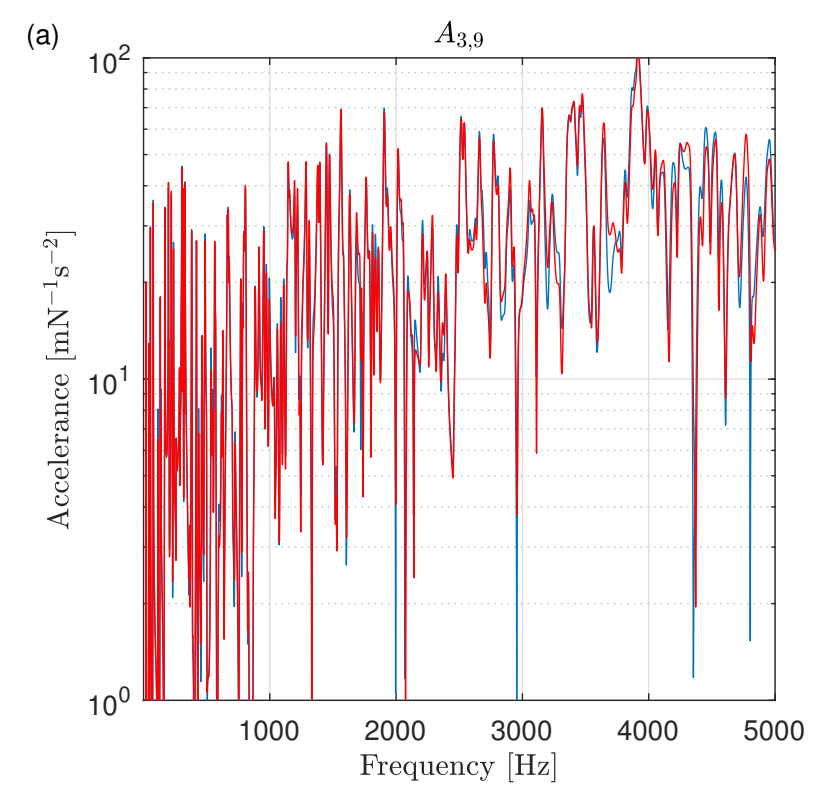

Figure 17 compares (a) the modulus of a component of both accelerance matrices (collocated and noncollocated) and (b) the modulus of a component of their respective apparent mass matrices. The results show that non-collocation has little effect on the accelerance obtained. However, huge differences can be observed in the apparent mass. The non-collocated FRF shows a large number of unexpected sharp spikes, and the bandwidth of these spikes is unaffected by the excitation frequency considered.

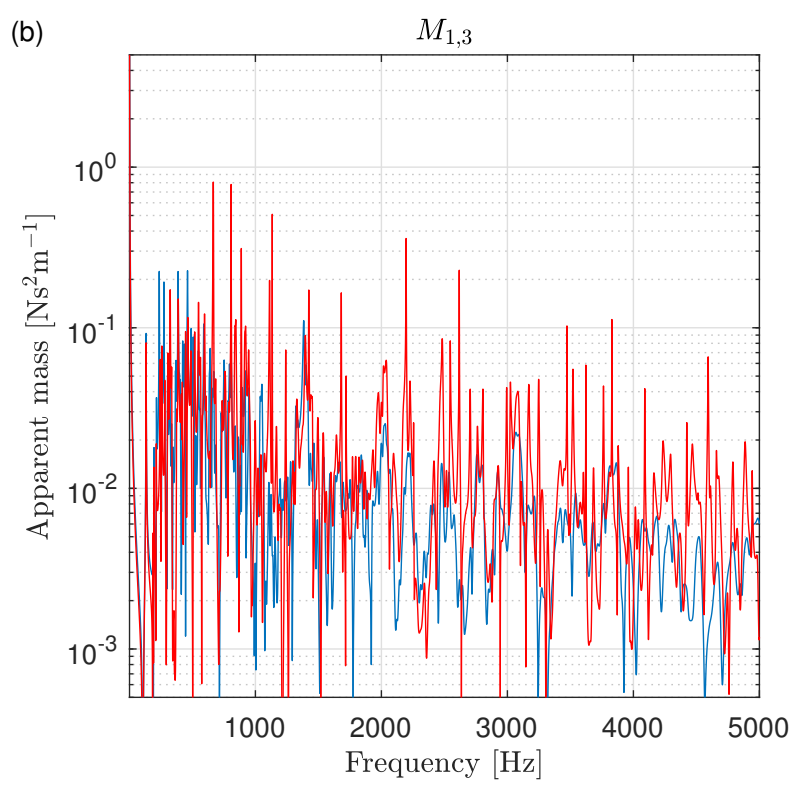

Figure 17: Results obtained using the analytical model for a lightly damped plate. (a) Modulus of the component (3,9) of the simulated collocated (blue) and non-collocated (red) accelerance matrices. (b) Modulus of the component (1,3) of the corresponding collocated (blue) and non-collocated (red) apparent mass matrices. 


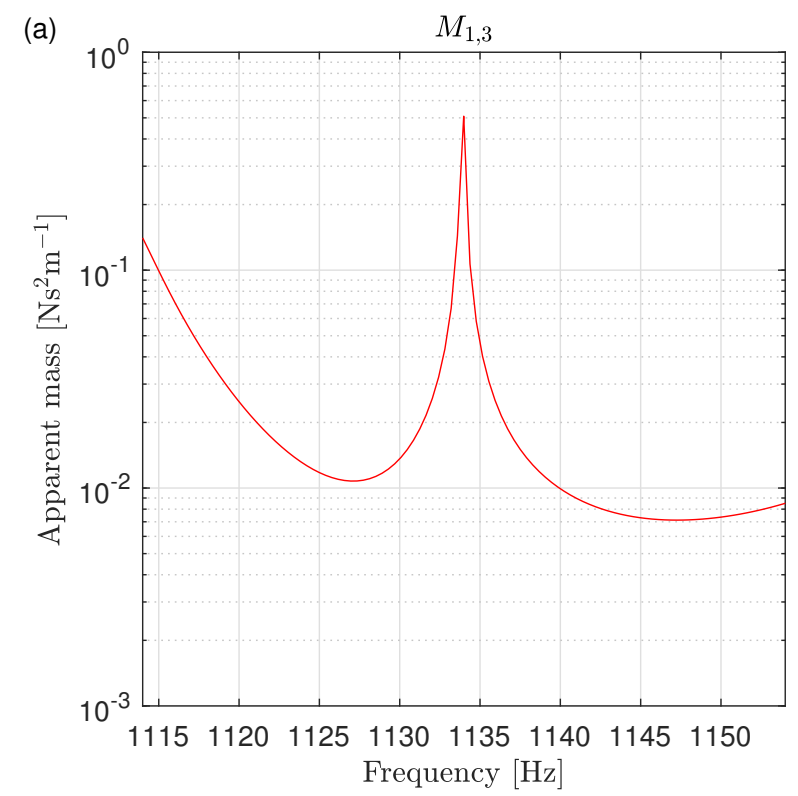

The non-collocated results presented in Figure 17 seem to be consistent with the experimental results presented in Figure 14. The consistency between the experimental results and the analytical simulations can be also observed in Figure 18, which presents an expanded view of one of the spikes of the non-collocated apparent mass component, and the frequency content of the accelerance matrix determinant and adjoint. As in Figure 15, the spike occurrence is related to a sharp decay in the determinant value.

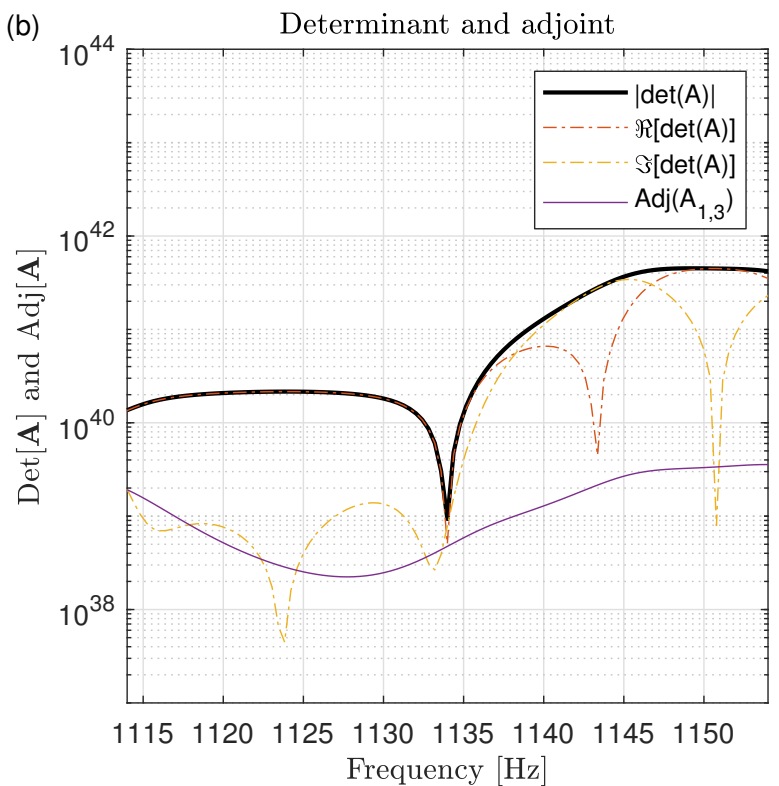

Figure 18: (a) Zoomed view of one of the spikes observed in the apparent mass matrix component shown in Figure 17 (b) Modulus of the terms involved in calculating the non-collocated apparent mass matrix. black: determinant; dashed blue: real component of the determinant; dashed red: imaginary part of the determinant, yellow: adjoint.

If an excitation vector $\mathbf{f}$ is applied at the force positions, the resulting acceleration vector a (i.e the response at the receiver positions) will be given by $\mathbf{a}=\mathbf{A f}, \mathbf{A}$ being the considered accelerance matrix. The results presented in Figure 18 show that an unexpected spike in the apparent mass is related to a sharp decay in the non-collocated accelerance matrix $\mathbf{A}_{\mathrm{nc}}$ determinant. Therefore, each of these peaks where $\operatorname{det}\left(\mathbf{A}_{\mathrm{nc}}\right) \rightarrow 0$, can be associated with a force eigenvector. Then, if one of these force eigenvectors is multiplied by $\mathbf{A}_{\mathrm{nc}}$, the receiver positions should not be accelerated. However, if that same force eigenvector is multiplied by the corresponding collocated accelerance matrix $\mathbf{A}_{\mathrm{c}}$ the receiver positions will move. This result has been verified by applying the force eigenvector associated with the spike observed in Figure 18 to (a) the force positions $\left(x_{f_{k}}, y_{f_{k}}\right)$ considered in the non-collocated case, and (b) to the force positions considered in the collocated one. The response of the plate to these excitations is presented in Figure 19. 


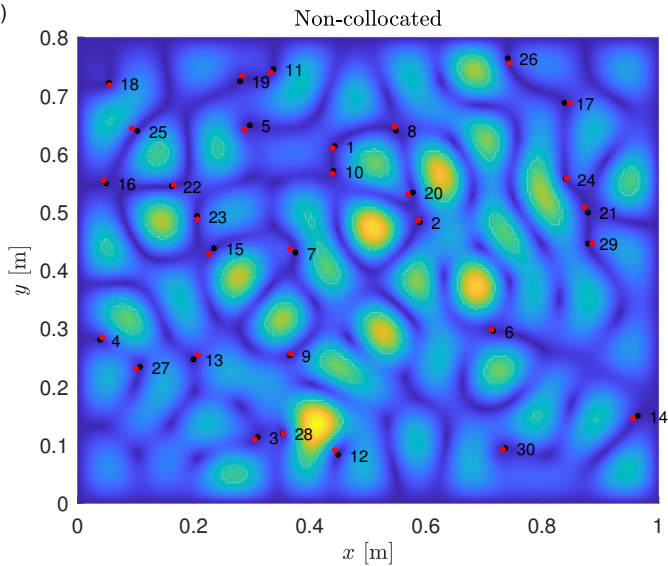

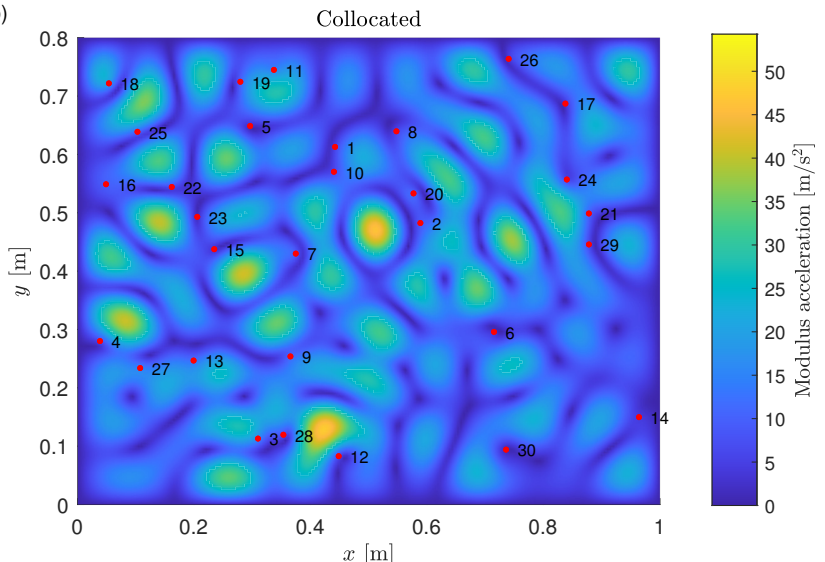

Figure 19: Modulus of the plate acceleration when it is excited by the force eigenvector corresponding to the spike presented in Figure 18 (a) Force positions are non-collocated (b) Force positions are collocated.

The results show that a small change in the force positions can have a huge effect on the plate response field. For the non-collocated case, the results also indicate that the response at the receiver positions (marked in this case using red dots) is almost zero. This result ensures that the applied force vector is in fact a force eigenvector, and verifies that a sharp peak in the apparent mass matrix can be related to an eigenvalue problem.

The lack of unexpected spikes for the collocated case can be justified noting that, when the force and receiver points are the same, a force vector giving $\mathbf{A}_{c} \mathbf{f}=\mathbf{0}$ would be a blocked force vector. If this blocked force existed and was applied to the receiver positions, it would give a zero input power to the system. This situation would not be consistent with the principle of conservation of energy, due to the fact that energy will be dissipated due to the plate's damping. Therefore, the type of spikes observed the non-collocated case cannot happen in this case.

A near-zero response at multiple positions would be also compatible with the unexpected case where these positions lie in nodal lines of the particular mode shape that dominates the response at the considered excitation frequency. However, a more detailed examination of contribution that each plate mode has on the plate response has shown that (i) the unexpected spikes in the apparent mass matrix occur at frequencies that are not related to the plate eigenfrequencies, and (ii) the response of the plate at these frequencies includes the contribution of a large number of modes.

Further insights into the conditions in which unexpected spikes occur can be obtained if the determinant of the non-collocated accelerance matrix is expressed as $\operatorname{det}\left|\mathbf{A}_{\mathrm{nc}}\right|=\prod_{i=1}^{N} \lambda_{i}$, being $\lambda_{i}$ the (complex) matrix eigenvalues. This product of eigenvalues suggests that it is sufficient to have one $\left|\lambda_{i}\right|=0$ to find a spike in the apparent mass matrix. However, such condition implies that both real and imaginary components of the eigenvector vanish. As the complex component of an off-resonance response is small for low damping 
(a)

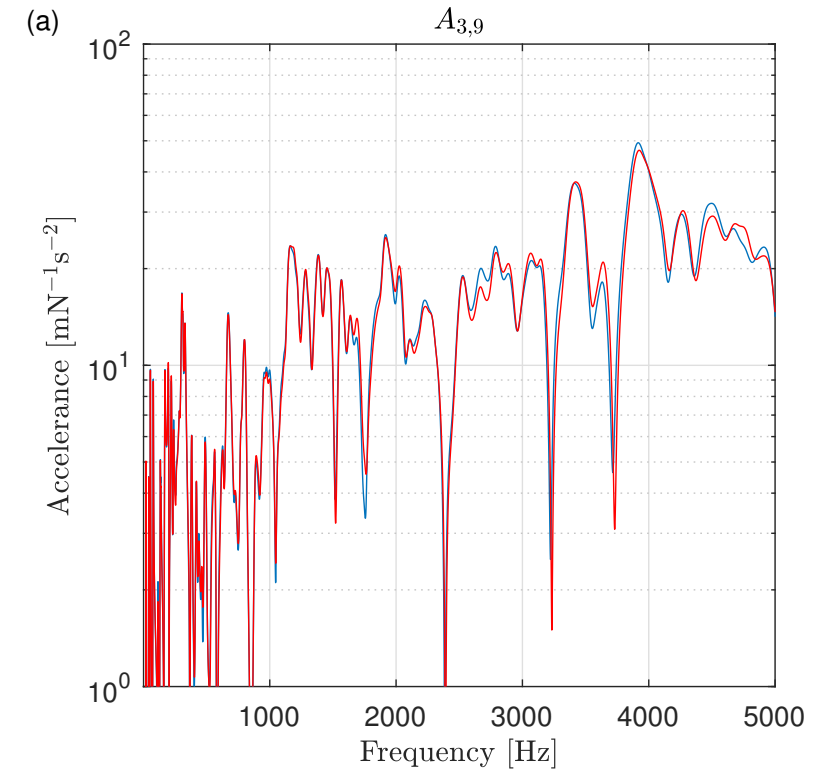
lightly damped one. consistent with the previous discussion. (b)

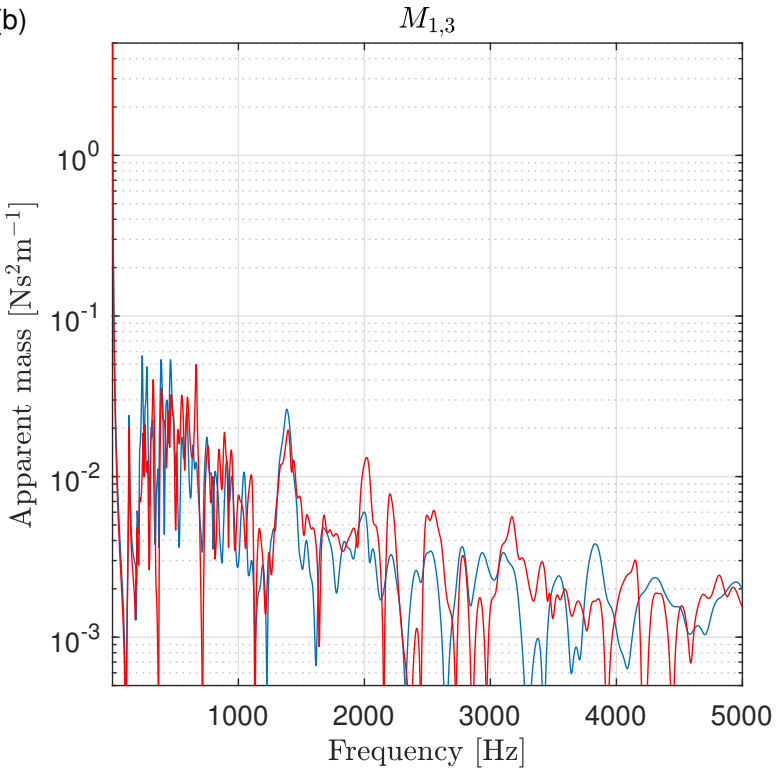

Figure 20: Results obtained using the analytical model for a heavily damped plate. (a) Modulus of the component (3,9) of the simulated collocated (blue) and non-collocated (red) accelerance matrices. (b) Modulus of the component (1,3) of the corresponding collocated (blue) and non-collocated (red) apparent mass matrices.

values, it is expected that a zero eigenvalue, and therefore a spike in the apparent mass matrix, would be less likely to happen if the measurements are performed with a heavily damped system rather than with a

This result has been verified in Figure 20, where components of the collocated and non-collocated accelerance and apparent mass matrices are compared for a heavily damped $(\eta=3.2 \%)$ plate. The results show that the non-collocated apparent mass matrix does not have any unexpected spikes, a result that is

b) Modulus of the component $(1,3)$ of the

\subsubsection{Plate with added damping}

A second artificial ensemble of thin rectangular plates has been obtained using the plate with an added damping treatment (see Figure 1b). As in the previous case, the response of the damped plate to hammer impacts was measured at the 30 positions marked with dots in Figure 10b. The same $N_{I}=9$ positions of interest have been considered and, for each member of the ensemble, point masses of $70 \mathrm{~g}$ have been numerically added at 11 positions that are randomly chosen from the $N_{P}=30-N_{I}=21$ positions. Again, the method has been used to build a 20 member ensemble.

Figure 21] shows the modulus of a component of the initial accelerance matrix $\mathbf{A}_{\text {ini }}$ and of the initial apparent mass matrix $\mathbf{M}_{\text {ini }}$ for the plate with added damping. When compared to the case without added damping (see Figure 14), it is clear that the apparent mass matrix for this case presents far fewer unexpected spikes. This reduction is consistent with the discussion of the results obtained using the analytical plate 
model.
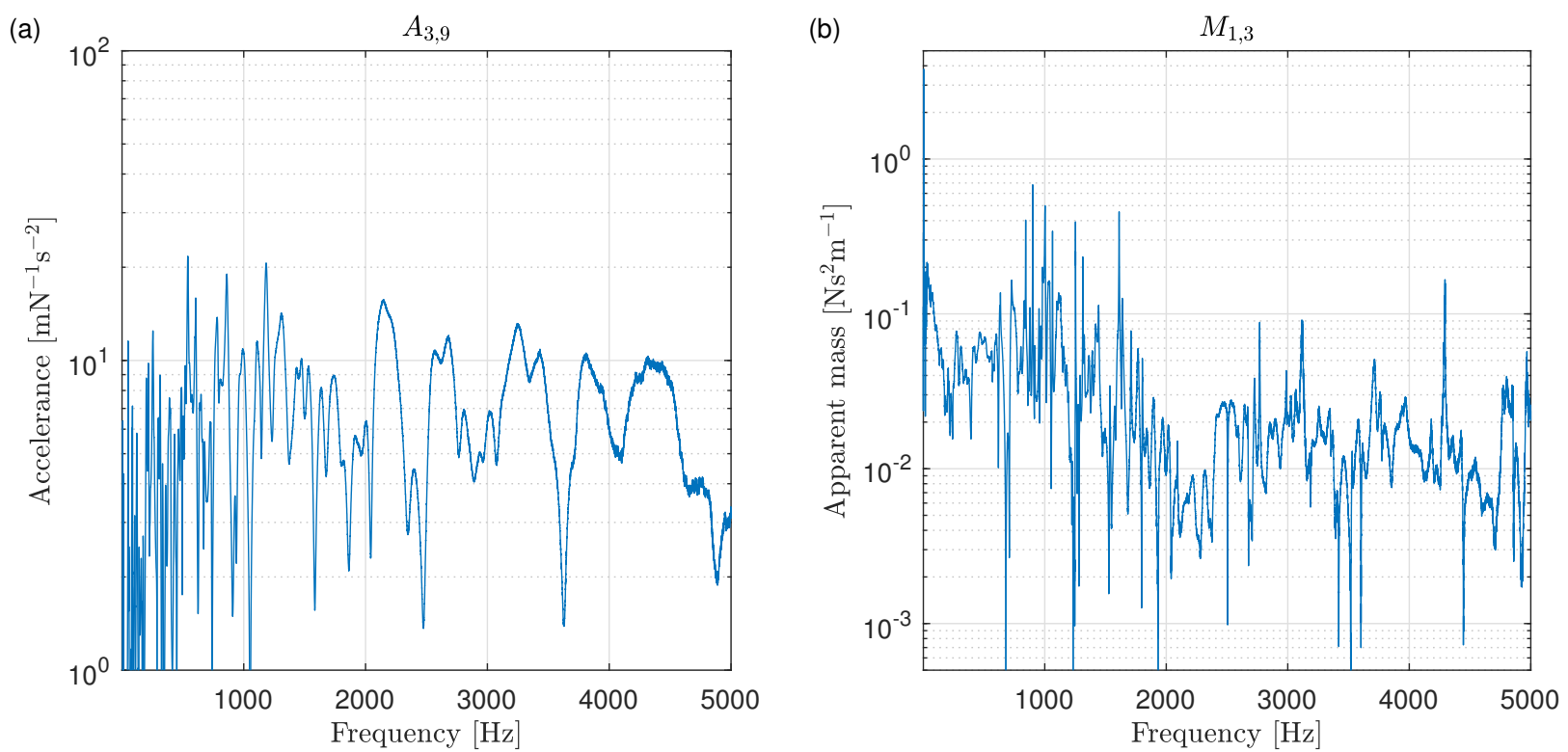

Figure 21: (a) Modulus of the component (3,9) of the initial accelerance matrix $\mathbf{A}_{\text {ini }}$ for the plate with added damping. (b) Modulus of the component (1,3) of the initial apparent mass matrix $\mathbf{M}_{\text {ini }}$ for the plate with added damping.

The statistics of both experimental and artificial ensembles have been again compared to SEA mean and variance predictions. As in the case of the plate without added damping, the vibration energy of the plate has been estimated using $\mathrm{E}[E]=m_{\mathrm{p}}\left\langle|v|^{2}\right\rangle_{\mathrm{a}} / 2$. As before, for the experimental ensemble case, the space average considers two of the interior points when the third one is excited and, for the artificial ensemble one, the average uses five of the interior positions when the sixth one is excited.

Figure 22 compares the ensemble mean and relative variance of the estimated experimental vibrational energy of the plate with the SEA ensemble mean and relative variance predictions, calculated using Eqs. (13) and (16), respectively. These predictions have been calculated using the experimentally determined loss factor for the damped plate, defined in Section 2. The energy predicted for each one of the 20 members of the ensemble has been also included in the results. 

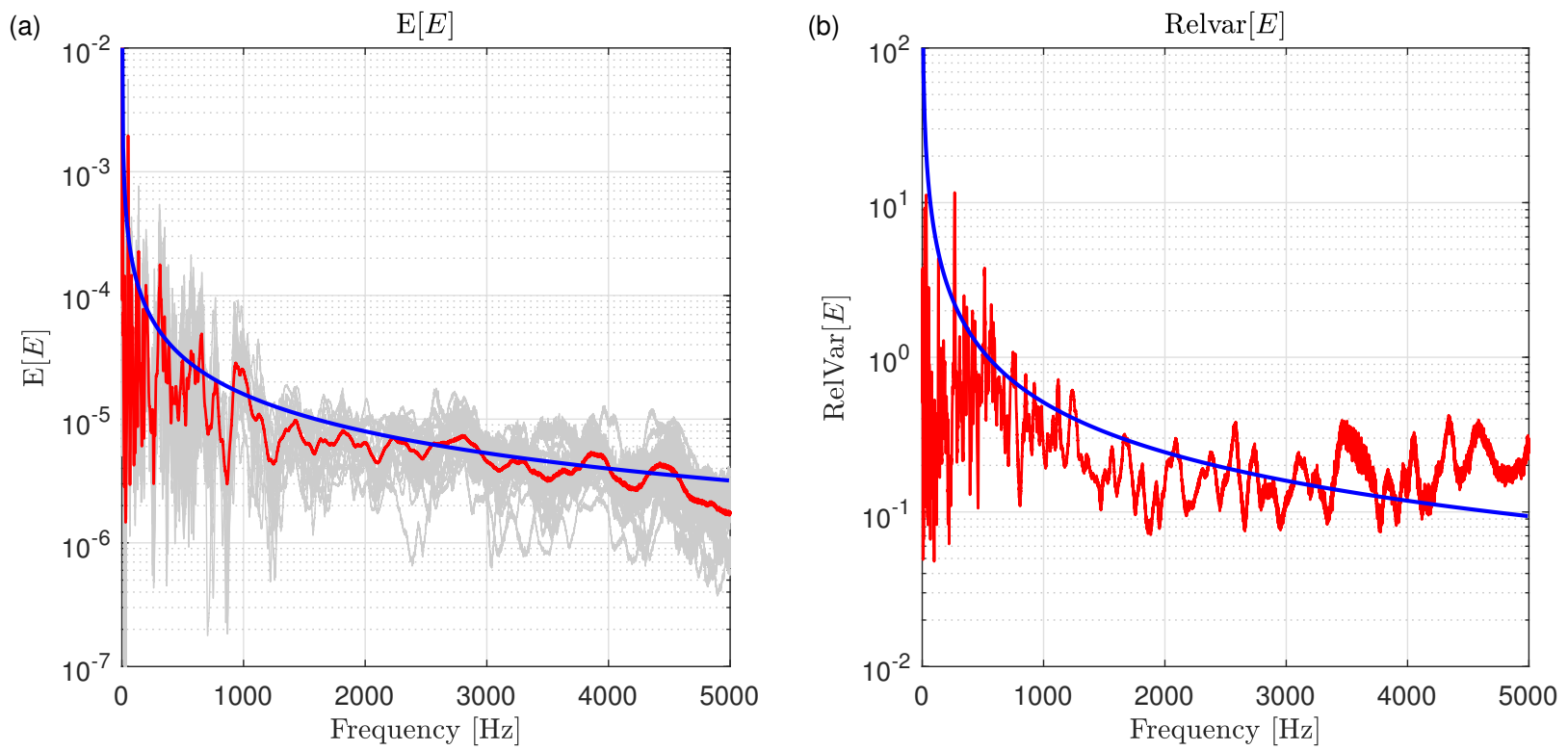

Figure 22: (a) Energy of the plate with added damping due to a unit point force excitation. Gray: response of the 20 members of the experimental ensemble; red: experimental ensemble mean response; blue: SEA prediction. (b) Relative variance of the energy. Red: experimental ensemble variance; blue: SEA prediction.

The results show that, despite having used only two (near) positions to estimate the plate energy, the statistics of the experimental ensemble agree well with the theoretical predictions.

The same comparison has been performed in Figure 23 using the artificially generated ensemble. The results are slightly better than the ones for the plate without added damping (Figure 13) but, specially in the relative variance case, the results are still quite noisy. Despite that, it can be concluded that the experimental issues found in the generation of artificial ensembles mainly occur due to a low damping value in the considered subsystem. 

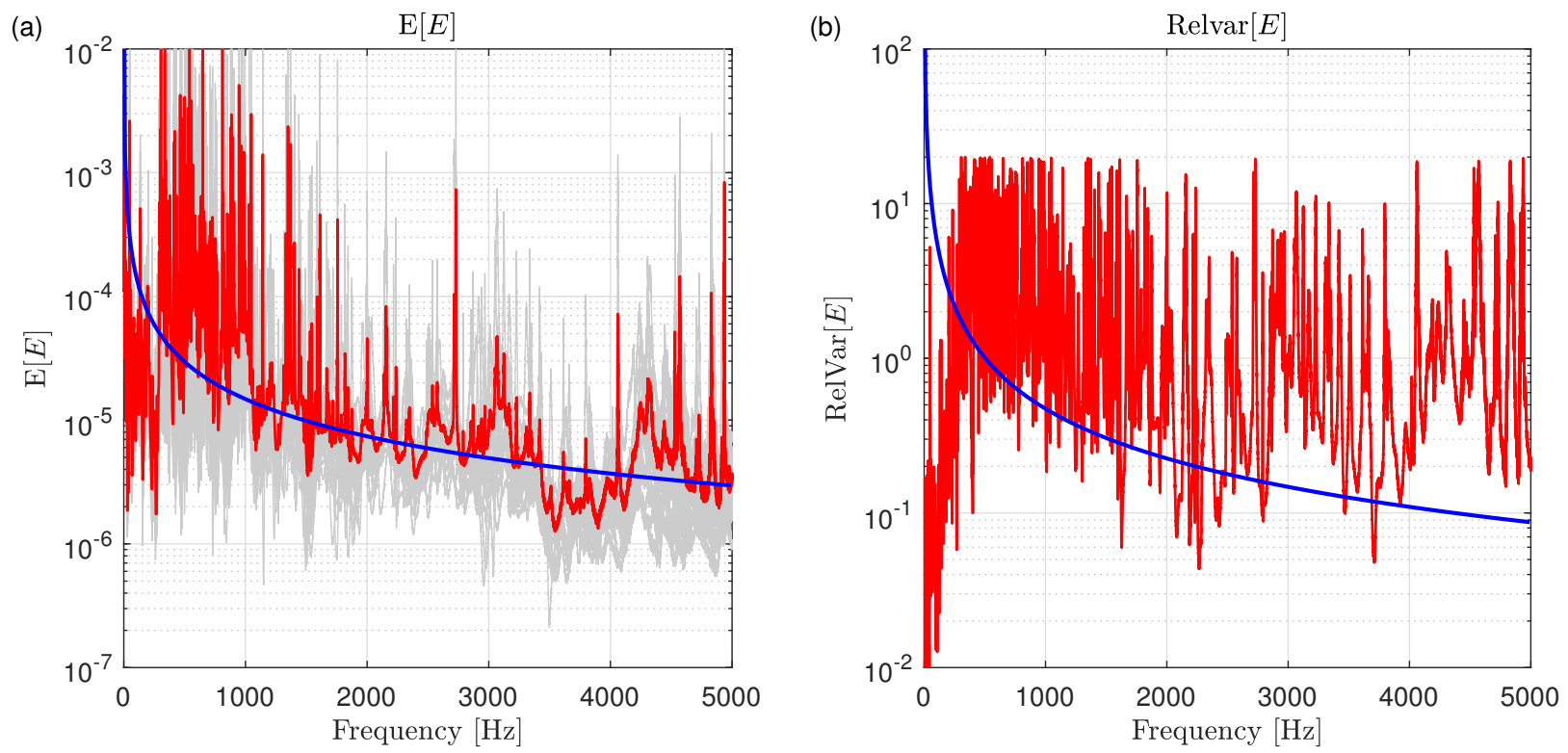

Figure 23: (a) Energy of the plate with added damping due to a unit point force excitation. Gray: response of the 20 members of the artificial ensemble; red: artificial ensemble mean response; blue: SEA prediction. (b) Relative variance of the energy. Red: artificial ensemble variance; blue: SEA prediction.

The results presented in Figure 23 show that an artificial ensemble may be of limited use in determining the energy statistics of a subsystem having uncertainties. However, this type of ensemble could also be used to obtain another result of interest: a direct field dynamic stiffness matrices. This potential application is studied in Figure 24, in which dynamic stiffness ensemble averages are used to determine $D_{\text {dir }}$ for a point far from any of the plate's edges, referred as interior point, and for a point lying near one of these edges, referred as near edge point. Both experimental and artificial ensemble averages are compared with the analytical expressions for $D_{\text {dir }}$, which are computed, for the interior point case, using Eq. (6) and, for the near edge one, using the methodology presented in Appendix A. As in Subsection 3.2, both experimental dynamic stiffness are obtained using Eq. (7). As in previous cases, the experimental results have been obtained using the inverse of the ensemble average instead of the ensemble average of the inverse. 

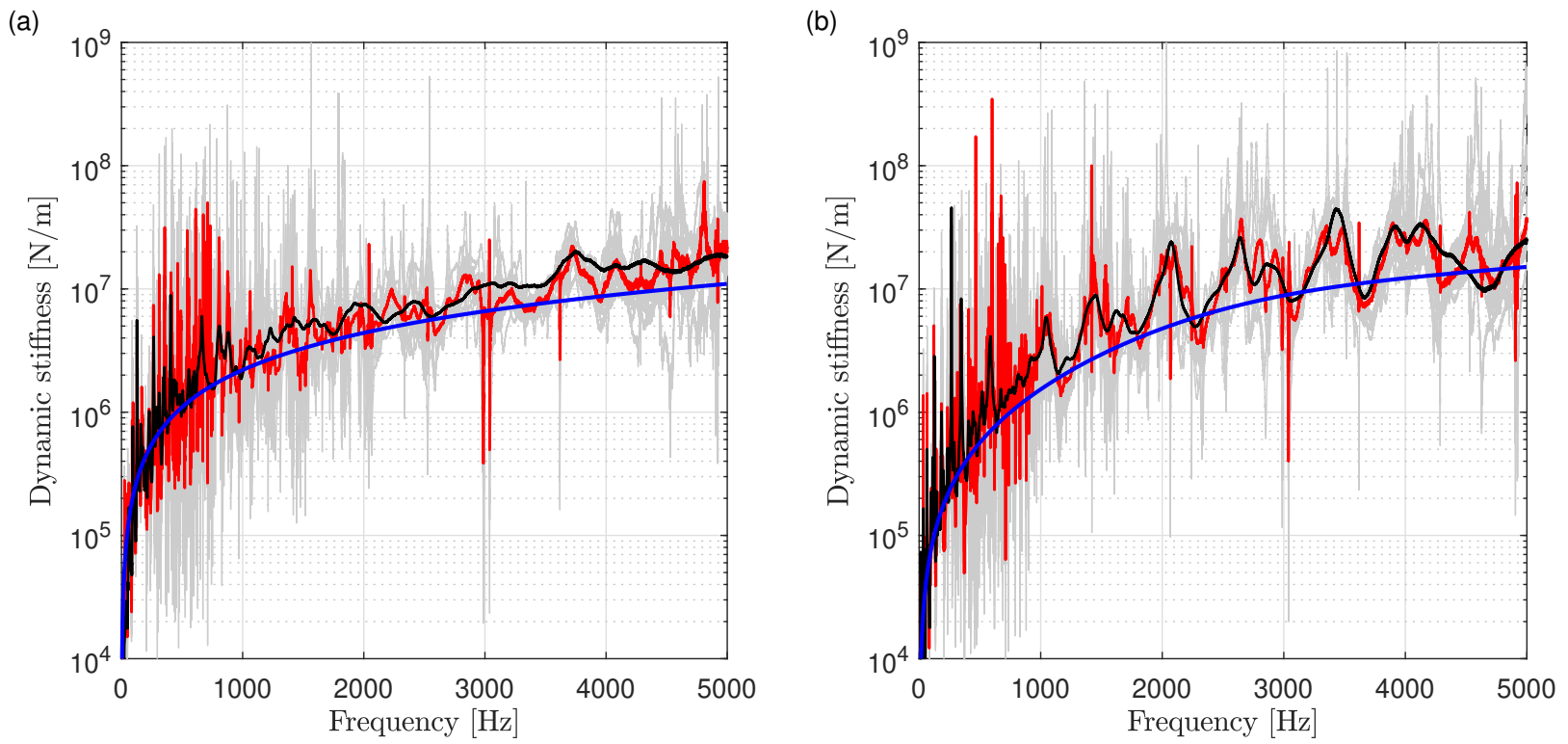

Figure 24: Direct field dynamic stiffness. Black: Experimental ensemble mean; red: Artificial ensemble mean; thick blue: analytical expression; gray: Dynamic stiffness of the 20 members of the artificial ensemble. (a) Interior point. (b) Near edge point.

The results show a reasonably good agreement between both ensemble averages and the analytical predictions. The predicted stiffness is slightly higher than the experimental results, a result that can be attributed to a local effect of the applied damping treatment (see Figure 1b). As before, the artificial ensemble results are nosier than the experimental ones.

One of the key advantages of considering an ensemble generated by randomising virtual masses instead of physical ones is that, once the initial accelerance matrix has been determined, large ensembles can be generated with very little effort. This advantage has been used in Figure 25 to investigate if the use of a larger ensemble can improve the agreement between artificial and experimental ensemble averages. In particular, an artificial ensemble of 1280 members has been used in the figure. 
(a)

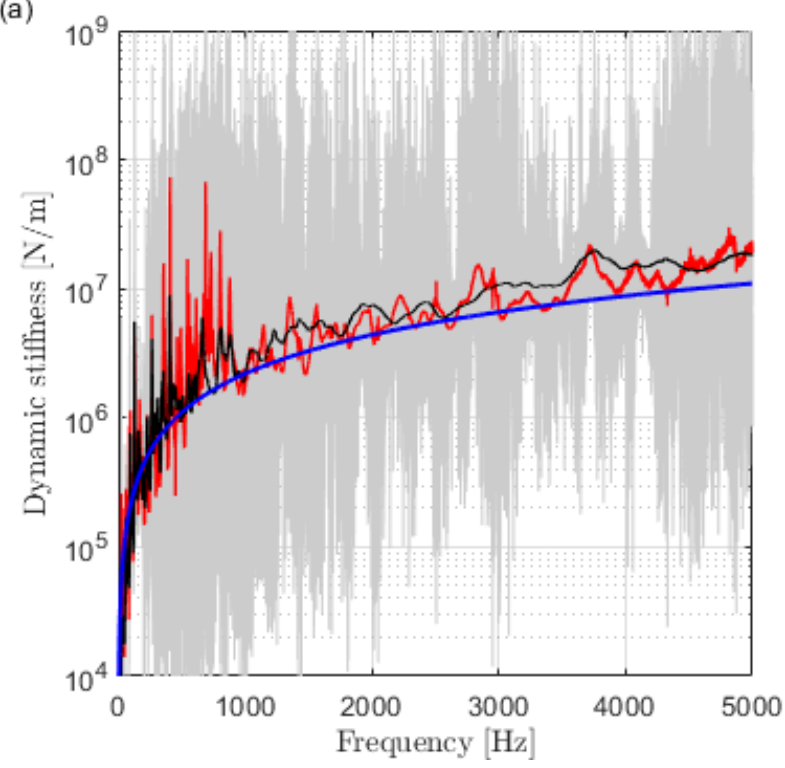

(b)

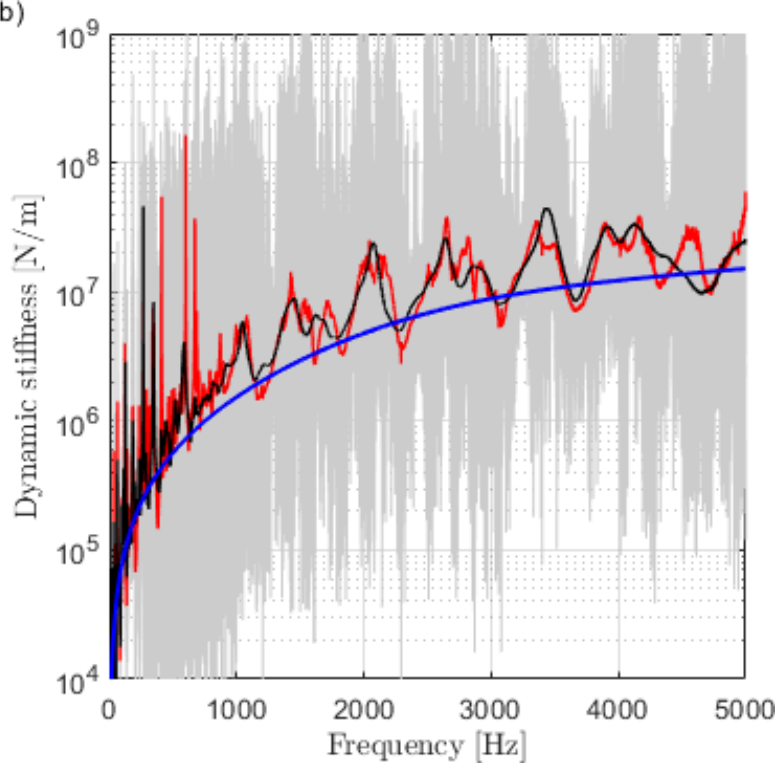

Figure 25: Direct field dynamic stiffness. Black: Experimental ensemble mean; red: Artificial ensemble mean; thick blue: analytical expression; gray: Dynamic stiffness of the 1280 members of the artificial ensemble. (a) Interior point. (b) Near edge point.

The results show that the new artificial ensemble average agrees very well with the experimental one. The unexpected high-frequency spikes have been clearly smoothed in this case. However, the huge statistical spread of the response also suggests that a larger ensemble would not improve the variance results presented in Figure 23. Additional calculations have confirmed this result.

This section has shown that ensembles generated using virtual point masses have potential advantages over ensembles generated by physically randomising the system of interest. However, the applicability of these artificial ensembles may be limited to those cases where the system is significantly damped.

\section{Conclusions}

This work has presented an experimental study of certain fundamental properties of random causal frequency response functions. This exploration has been performed by measuring the dynamic response of an ensemble of random plates. Two experimental ensembles have been obtained by physically randomising the considered structure with and without an added damping treatment.

The experimental results have been initially used to verify that ensembles of measured accelerances satisfy the analyticity-ergodicity condition. This result represents a first experimental validation of a property that has been recently demonstrated to be applicable to random engineering systems.

The ensembles have been also used to demonstrate that the direct field dynamic stiffness of a junction between systems, a key parameter in the hybrid FE-SEA method, can be determined using experimental 
data. This property has been successfully applied to three types of point connections of increasing complexity.

A methodology to generate an ensemble of random system using virtual masses has been proposed for those cases where an experimental randomisation is impractical. The results, however, have shown that for lightly damped systems this methodology is extremely sensitive to small experimental imprecisions. The effect of these small imprecisions was further discussed using an analytical model, and the results have shown that the experimental issues are clearly reduced when a system is heavily damped. Experimental results for a plate with added damping treatment has supported this numerical result.

\section{Acknowledgements}

The authors gratefully acknowledge the financial support provided by the Engineering and Physical Sciences Research Council under grant number EP/P005489/1, Design by Science, with industrial partners Bentley Motors Ltd, Brüel \& Kjær, Dyson Ltd and Wave six LLC. The authors would also like to express their gratitude to Dr David Hawes for his contribution to the near edge point calculations presented in this work.

\section{Appendix A. Modelling a near edge point}

\section{Appendix A.1. Proposed numerical approach}

This appendix presents a numerical strategy to compute the dynamic stiffness associated with the transverse response of a point connection close to the edge of a semi-infinite plate. The first step of the approach consists of dividing the initial structure, presented in Figure A.1a, into an infinite strip with a thickness equal to the distance point-edge distance, and a semi-infinite plate in which the point connection lies exactly on its edge, as shown in Figure A.1b

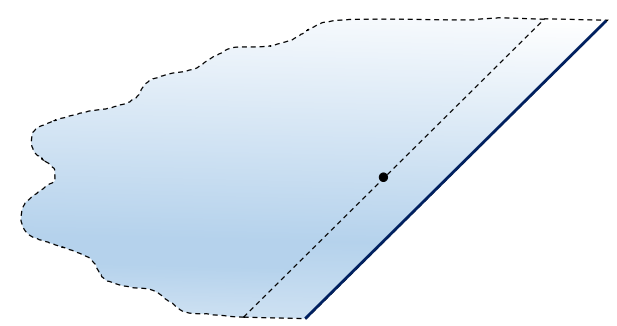

(a) Scheme of a point near the edge of a semiinfinite plate.

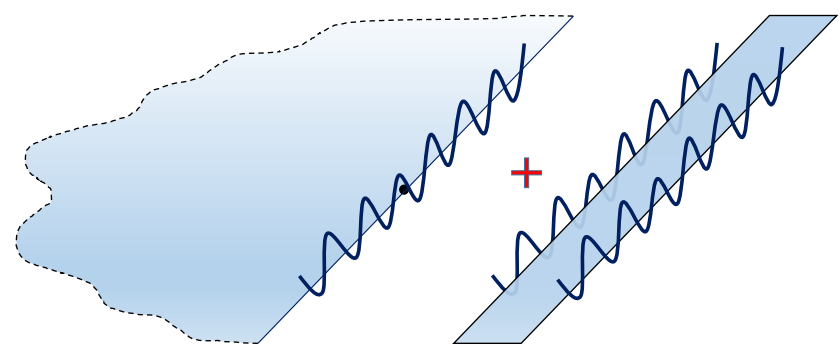

(b) Substructuring approach used to compute the point stiffness for a point near the edge of a semi-infinite plate.

Figure A.1: Method used to compute $D_{\text {dir }}$ for a point near a plate's edge. 
Assuming plane wave propagation, the dynamic stiffness of the original structure along the dividing line, noted as $\mathbf{D}_{\text {near }}(k, \omega)$, can be expressed as

$$
\mathbf{D}_{\text {near }}(k, \omega)=\mathbf{D}_{\mathrm{e}}(k, \omega)+\mathbf{D}_{\text {strip }}(k, \omega)
$$

where $\mathbf{D}_{\mathrm{e}}(k, \omega)$ is the dynamic stiffness along the edge of a semi-infinite plate, and $\mathbf{D}_{\text {strip }}(k, \omega)$ is dynamic stiffness along the edge of an infinite plate strip. The wavenumber and frequency dependence $(k, \omega)$ has been explicitly written, but will be omitted for brevity in what follows.

The dynamic stiffness along the edge of a semi-infinite plate $\mathbf{D}_{\mathrm{e}}(k, \omega)$ can be computed using the formulation presented in [6]. Assuming plane wave propagation, the out-of-plane displacement $w$ and rotation $\theta$ of the plate's edge caused by edge tractions $S$ and $M$ can be expressed as

$$
\mathbf{D}_{e}\left(\begin{array}{l}
w \\
\theta
\end{array}\right)=\left(\begin{array}{c}
S \\
M
\end{array}\right)
$$

where the components of the dynamic stiffness matrix $\mathbf{D}_{e}$ can be found in [29].

The dynamic stiffness of the edges of an infinite strip plate can be obtained extending the formulation presented in [30, which considers a finite plate strip simply-supported on its narrow sides, to the infinite case. The formulation is extended by assuming a continuous set of wavenumbers $k$ instead of a discrete one. Assuming plane wave propagation, the out-of-plane response of both strip edges due to edge tractions can be expressed as

$$
\left(\begin{array}{c}
-S_{l} \\
M_{l} \\
S_{r} \\
-M_{r}
\end{array}\right)=\left(\begin{array}{ll}
\mathbf{D}_{l l} & \mathbf{D}_{l r} \\
\mathbf{D}_{r l} & \mathbf{D}_{r r}
\end{array}\right)\left(\begin{array}{c}
w_{l} \\
w_{l}^{\prime} \\
w_{r} \\
-w_{r}^{\prime}
\end{array}\right)
$$

where the subindex $r$ refers to a "right edge" term, and $l$ to a "left edge" term, and where the expression of the block matrices can be found in [30]. Assuming free-boundary conditions on the right edge of the strip, i.e. $S_{r}=-M_{r}=0$, the left edge dynamic stiffness can be expressed as the following condensed dynamic stiffness matrix

$$
\mathbf{D}_{\text {strip }}=\mathbf{D}_{l l}-\mathbf{D}_{l r} \mathbf{D}_{r r}^{-1} \mathbf{D}_{r l}
$$

The receptance matrix along the dividing line $\mathbf{H}_{\text {near }}$ will be given by $\mathbf{H}_{\text {near }}=\mathbf{D}_{\text {near }}^{-1}$. The receptance of a point near the semi-infinite edge $H_{\mathrm{dp}}$ can be obtained by applying an inverse Fourier transform to the first component of $\mathbf{H}_{\text {near }}$ as follows

$$
H_{\mathrm{dp}}(\omega)=\frac{1}{2 \pi} \int_{-\infty}^{\infty} H_{\text {near }, 11}(k, \omega) \mathrm{d} k
$$


where the wavenumber and frequency dependencies have been added for clarity. Finally, the direct field dynamic stiffness for a near edge point connection will be given by $D_{\mathrm{dir}}(\omega)=1 / H_{\mathrm{dp}}(\omega)$.

\section{Appendix A.2. Numerical validation}

The validity of the proposed method is assessed in this subsection by comparing it to a FE approach. The comparison has been performed considering a point situated at $2.5 \mathrm{~cm}$ from one of the edges of a rectangular thin aluminium plate, with dimensions $1.52 \mathrm{~m}$ (length) $\times 0.95 \mathrm{~m}$ (width) $\times 2 \mathrm{~mm}$ (thickness). An ensemble of 20 systems has been obtained by randomising the numerical model using 10 point masses, each of them having $1 \%$ of the bare plate's mass. The model has been used to compute an ensemble of driving point responses for a dof representing the out-of-plane displacement of a node near one of the plate's edge. The corresponding FE direct field dynamic stiffness has been then computed using Eq. 5

Figure A.2 compares the (a) real and (b) imaginary components of the dynamic stiffness obtained using the proposed numerical approach with the one obtained ensemble averaging the FE results. Two loss factor values have been considered in the comparison: $\eta=0.004$ and $\eta=0.03$. A very good agreement between both methods has been found, ensuring that the proposed approach is a computationally efficient method for obtaining the direct field dynamic stiffness associated with the transverse response of a near-edge point in a thin plate.
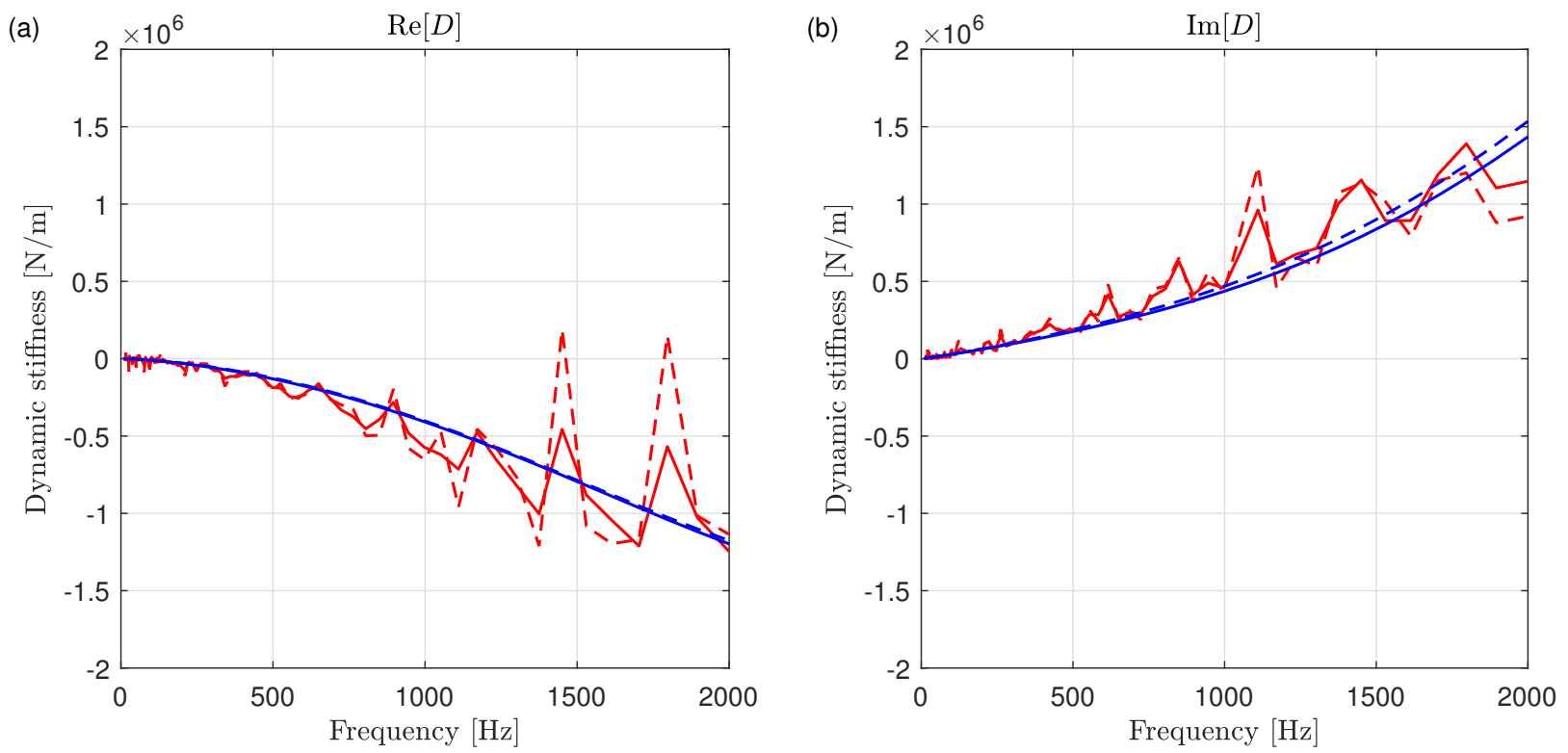

Figure A.2: Direct field dynamic stiffness for a single point connection near one plate edge. Red: FE ensemble mean with $\eta=0.03$; dashed red: FE ensemble mean with $\eta=0.004$; blue: wave-based calculation with $\eta=0.03$; dashed blue: wave-based calculation with $\eta=0.004$. (a) Real components. (b) Imaginary components. 


\section{References}

[1] R. Lyon, R. DeJong, Theory and Application of Statistical Energy Analysis, Butterworth-Heinemann, Boston, 1995.

[2] R. S. Langley, V. Cotoni, Response variance prediction in the statistical energy analysis of built-up systems, J. Acoust. Soc. Am. 115 (2) (2004) 706.

[3] P. Shorter, R. Langley, Vibro-acoustic analysis of complex systems, J. Sound Vib. 288 (3) (2005) 669-699.

[4] P. Shorter, R. Langley, On the reciprocity relationship between direct field radiation and diffuse reverberant loading, J. Acoust. Soc. Am. 117 (1) (2005) 85-95.

[5] R. Langley, On the diffuse field reciprocity relationship and vibrational energy variance in a random subsystem at high frequencies, J. Acoust. Soc. Am. 121 (2) (2007) 913-921.

[6] V. Cotoni, P. Shorter, R. Langley, Numerical and experimental validation of a hybrid finite element-statistical energy analysis method, J. Acoust. Soc. Am. 122 (1) (2007) 259-270.

[7] R. Langley, V. Cotoni, Response variance prediction for uncertain vibro-acoustic systems using a hybrid deterministicstatistical method., J. Acoust. Soc. Am. 122 (6) (2007) 3445-63.

[8] A. Cicirello, R. Langley, The vibro-acoustic analysis of built-up systems using a hybrid method with parametric and non-parametric uncertainties, J. Sound Vib. 332 (9) (2013) 2165-2178.

[9] A. Cicirello, R. S. Langley, Efficient parametric uncertainty analysis within the hybrid Finite Element/Statistical Energy Analysis method, J. Sound Vib. 333 (6) (2014) 1698-1717.

[10] A. Clot, J. Meggitt, R. Langley, A. Elliott, A. Moorhouse, Development of a hybrid fe-sea-experimental model, J. Sound Vib. 452 (2019) $112-131$

[11] A. Moorhouse, A. Elliott, T. Evans, In situ measurement of the blocked force of structure-borne sound sources, J. Sound Vib. 325 (4-5) (2009) 679-685.

[12] J. Meggitt, A. Elliott, A. Moorhouse, In-situ determination of dynamic stiffness for resilient elements, P. I. Mech. Eng. C-J. Mec. 230 (6) (2016) 986-993.

[13] R. S. Langley, P. J. Shorter, The wave transmission coefficients and coupling loss factors of point connected structures, J. Acoust. Soc. Am. 113 (4 Pt 1) (2003) 1947-1964.

[14] R. S. Langley, J. A. Cordioli, Hybrid deterministic-statistical analysis of vibro-acoustic systems with domain couplings on statistical components, J. Sound Vib. 321 (3-5) (2009) 893-912.

[15] M. S. Kompella, R. J. Bernhard, Measurement of the statistical variation of structural-acoustic characteristics of automotive vehicles, Tech. rep., SAE Technical Paper (1993).

[16] R. H. Lyon, Statistical Analysis of Power Injection and Response in Structures and Rooms, J. Acoust. Soc. Am. 45 (3) (1969) 545-565.

[17] R. H. Lyon, Progressive phase trends in multi-degree-of-freedom systems, J. Acoust. Soc. Am. 73 (4) (1983) $1223-1228$.

[18] E. J. Skudrzyk, Simple and complex vibratory systems, Pennsylvania State Univ Pr, 1968.

[19] L. Cremer, M. Heckl, E. Ungar, Structure-borne sound, Springer-Verlag, Berlin, 1985.

[20] R. S. Langley, On the statistical properties of random causal frequency response functions, J. Sound Vib. 361 (2016) $159-175$.

[21] P. A. Mello, P. Pereyra, T. H. Seligman, Information theory and statistical nuclear reactions. i. general theory and applications to few-channel problems, Ann. Phys.-New York 161 (2) (1985) 254-275.

[22] T. Brody, J. Flores, J. B. French, P. A. Mello, A. Pandey, S. S. M. Wong, Random-matrix physics: spectrum and strength fluctuations, Rev. Mod. Phys. 53 (3) (1981) 385.

[23] A. Nock, S. Kumar, H.-J. Sommers, T. Guhr, Distributions of off-diagonal scattering matrix elements: Exact results, Ann. Phys.-New York 342 (2014) 103-132. 
[24] R. Langley, A. Brown, The ensemble statistics of the energy of a random system subjected to harmonic excitation, J. Sound Vib. 275 (3-5) (2004) 823-846.

[25] D. J. Ewins, Modal testing: theory and practice, Vol. 15, Research studies press Letchworth, 1984.

[26] A. T. Moorhouse, A. S. Elliott, The "round trip" theory for reconstruction of Green's functions at passive locations, J. Acoust. Soc. Am. 134 (5) (2013) 3605-3612.

[27] M. L. Mehta, Random matrices, Elsevier, 2004.

[28] R. L. Weaver, Spectral statistics in elastodynamics, J. Acoust. Soc. Am. 85 (3) (1989) 1005-1013.

[29] R. S. Langley, K. H. Heron, Elastic wave transmission through plate/beam junctions, J. Sound Vib. 143 (2) (1990) $241-253$.

[30] R. Langley, Application of the dynamic stiffness method to the free and forced vibrations of aircraft panels, J. Sound Vib. 135 (2) (1989) $319-331$. 